\title{
BRAIDED HOCHSCHILD COHOMOLOGY AND HOPF ACTIONS
}

\author{
CRIS NEGRON
}

\begin{abstract}
We show that the braided Hochschild cohomology, of an algebra in a suitably algebraic braided monoidal category, admits a graded ring structure under which it is braided commutative. We then give a canonical identification between the usual Hochschild cohomology ring of a smash product and the (derived) invariants of its braided Hochschild cohomology ring. We apply our results to identify the associative formal deformation theory of a smash product with its formal deformation theory as a module algebra over the given Hopf algebra (when the Hopf algebra is sufficiently semisimple). As a second application we deduce some structural results for the usual Hochschild cohomology of a smash product, and discuss specific implications for finite group actions on smooth affine schemes.
\end{abstract}

\section{INTRODUCTION}

Let $k$ be a field and $\mathscr{Z}$ be the category of modules over a quasitriangular Hopf algebra $H$ (see 2.2). This gives $\mathscr{Z}$ the structure of a braided monoidal category with a monoidal embedding into the category of vector spaces. Our motivating example is the category $Y D_{E}^{E}$ of Yetter-Drinfeld modules over a finite dimensional Hopf algebra $E$.

An algebra in $\mathscr{Z}$ will be an object $B$ in $\mathscr{Z}$ equipped with a compatible algebra structure. That is, we require all the structure maps

$$
\text { unit }: k \rightarrow B \text { and mult }: B \otimes B \rightarrow B
$$

to be maps in $\mathscr{Z}$. This is equivalent to $B$ being a $H$-module algebra when we specify $\mathscr{Z}=H \bmod$. Our primary example of such an algebra is a smash product $B=A * E$, where $E$ is a finite dimensional Hopf algebra acting on $A$. The smash product is an algebra in the category of Yetter-Drinfeld modules over $E$ under the right adjoint action (see 2.3).

The work of the present paper is motivated, in part, by a desire to understand the nature of the Hochschild cohomology of $A$ with coefficients in the smash product $H H^{\bullet}(A, A * E)$. This cohomology is, in a sense, more fundamental than the usual Hochschild cohomology $H H^{\bullet}(A * E)$. This can be seen in many works on Hochschild, cyclic, and orbifold cohomology (e.g. $[2,5,29,30,14,27,3])$. As is explained below, we find that the cohomology $H H^{\bullet}(A, A * G)$ is actually the braided Hochschild cohomology of

This work was supported by NSF Postdoctoral fellowship DMS-1503147. 
the smash product, with coefficients in itself. This phenomenon was first noticed by Schedler and Witherspoon in a more restrictive setting [28].

In [1] Baez considered the braided Hochschild homology of such an algebra $B$. He defined this homology as the usual Tor group of the corresponding braided enveloping algebra $B^{\underline{e}}$,

$$
H_{\bullet}^{c}(B)=\operatorname{Tor}_{\bullet}^{\mathrm{B}}(B, B) .
$$

Here $c$ denotes the braiding transformation on $\mathscr{Z}$. (One can see Section 3 for a precise definition of the braided enveloping algebra.) Baez related this homology to $c$-commutative differential forms on $B$, when $B$ is braided commutative. Baez's results are referential to the usual Hochschild homology of a commutative algebra, in which case one finds Khäler differentials in degree 1.

In this work we consider the braided Hochschild cohomology, which can be defined succinctly as the group of self extensions of $B$ over its braided enveloping algebra. We follow Baez's notation and take

$$
H_{c}^{\bullet}(B):=\operatorname{Ext}_{B \underline{e}}^{\bullet}(B, B) \text {. }
$$

We also consider a relative version of the cohomology $H_{c, E}^{\bullet}(B)$, which is defined for any subalgebra $E \subset B$ in $\mathscr{Z}$.

This work is concerned primarily with a graded ring structure on braided Hochschild cohomology and its relation(s) to the non-braided Hochschild cohomology of smash products, although there are also interactions between the braided Hochschild cohomology and the Gerstenhaber bracket [24, Ch. $3]$.

Theorem 1.1 (=5.11). Let $B$ be an algebra in a braided monoidal category $\mathscr{Z}$ as above.

(1) Each cohomology group $H_{c}^{i}(B)$ is itself an object in $\mathscr{Z}$.

(2) The cohomology $H_{c}^{\bullet}(B)$ admits a graded ring structure under which it is braided commutative.

The two above statements also hold for any relative cohomology $H_{c, E}^{\bullet}(B)$.

Theorem 1.1 is a quantum analog of the well known fact that the usual Hochschild cohomology of any algebra is a (graded) commutative ring [9]. We also prove

Theorem $1.2(=4.6 / 5.5)$. Let $E$ be a finite dimensional Hopf algebra and $A$ be an E-module algebra. There is an algebra isomorphism between the Hochschild cohomology $H H^{\bullet}(A, A * E)$ and the E-relative braided Hochschild cohomology $H_{c, E}^{\bullet}(A * E)$. When, further, $E$ is semisimple and cosemisimple the Hochschild cohomology $H^{\bullet}(A, A * E)$ is isomorphic to the non-relative braided cohomology $H_{c}^{\bullet}(A * E)$.

Theorem 1.2 was first proved by Schedler and Witherspoon in the case of a finite group acting on an algebra in characteristic 0 [28]. We also give a version of Theorem 1.2 for certain crossed products and twists of 
smash products (see 4.2). Theorem 1.2 implies that there is an algebra identification between $H H^{\bullet}(A * E)$ and the $E$-invariant classes in $H_{c}^{\bullet}(A * E)$ when $E$ is semisimple and cosemisimple, among other things (see Corollary 5.5). The theorem also implies that the cohomology $H H^{\bullet}(A, A * E)$ takes values in the category of Yetter-Drinfeld modules over $E$, and that it is braided commutative in that category.

The fact that, when $E$ is semisimple and cosemisimple, we have an isomorphism with the non-relative cohomology $H_{c}^{\bullet}(A * E)$ is of some significance. For example, we can use this fact to show that the formal deformation theory of $A * E$ as an associate algebra is equivalent to the formal deformation theory of $A * E$ as an $E$-module algebra (under the adjoint action). This is done in Section 4.1.

We also apply the two above theorems to gain some general information about the Hochschild cohomology of smash products $A * G$, where $G$ is a finite group. As mentioned above, in characteristic 0 there is an algebra equality

$$
H H^{\bullet}(A * G)=H H^{\bullet}(A, A * G)^{G}\left(\text { and now } H H^{\bullet}(A * G)=H_{c}^{\bullet}(A * G)^{G}\right),
$$

and more generally a spectral sequence relating the two cohomologies. Whence we study the cohomology $H H^{\bullet}(A * G)$ by studying $H H^{\bullet}(A, A * G)$.

The cohomology $H H^{\bullet}(A, A * G)$ decomposes as a sum

$$
H H^{\bullet}(A, A * G)=\bigoplus_{g \in G} H H^{\bullet}(A, A g)
$$

and we get an action of the Hochschild cohomology $H H^{\bullet}(A)=H H^{\bullet}(A, A e)$ on each summand induced by the multiplication on $H H^{\bullet}(A, A * G)$. In certain examples, these $H H^{\bullet}(A)$-actions determine a great deal of the multiplicative structure on the entire cohomology [29, 27].

In Proposition 6.1, we apply Theorems 1.1 and 1.2 to show that each summand $H^{\bullet}(A, A g)$ is a symmetric bimodule over the Hochschild cohomology of $A$, and find explicit ideals in $H H^{\bullet}(A)$ annihilating each given summand. In the case of a finite group acting on a smooth affine $k$-scheme $X$, for example, our results imply that each cohomology $H H^{\bullet}(k[X], k[X] g)$ carries a canonical module structure over the algebra of polyvector fields $\wedge^{\bullet} T_{X^{g}}$ on the fixed space $X^{g}$ (see 6.1). This result is expected from its $C^{\infty}$ analog, which was studied in [27]. We expect that the ideals specified in Proposition 6.1 are in fact the entire annihilators of each $H H^{\bullet}(k[X], k[X] g)$ for a general smooth affine $G$-scheme $X$ (see again 6.1).

\section{Contents}

1. Introduction 1

2. Background 4

3. Braided Hochschild cohomology as a $\mathscr{Z}$-valued invariant 6

4. Identifications with standard Hochschild cohomology for smash products 
5. The cup product and braided commutativity of braided Hochschild cohomology

6. Consequences for smash products with group algebras 26

$\begin{array}{ll}\text { Acknowledgements } & 28\end{array}$

References

\section{BACKGROUND}

2.1. Conventions. Throughout this work $\mathscr{Z}$ will always denote a braided monoidal category which admits an equality $\mathscr{Z}=H \bmod$, for some quasitriangular Hopf algebra $H$. We also fix the Hopf algebra $H$ throughout. The invariants $(-)^{\mathscr{Z}}$ is defined abstractly as maps from the unit $(-)^{\mathscr{Z}}=$ $\operatorname{Hom}_{\mathscr{Z}}\left(\mathbf{1}_{\mathscr{Z}},-\right)$. This will agree with the usual invariants $(-)^{\mathscr{Z}}=(-)^{H}$. We let $c$ denote the braiding transformation on $\mathscr{Z}$.

A dot - generally denotes the action of $H$, while juxtaposition denotes multiplication in a ring. All Hopf algebras are assumed to have bijective antipode.

By $\otimes$ we mean $\otimes_{k}$. We use a simplified Sweedler's notation $\Delta(x)=x_{1} \otimes x_{2}$ to denote comultiplication in a coalgebra. So " $x_{1} \otimes x_{2}$ " here is a symbol denoting a sum of pure tensors.

Given an abelian category $\mathscr{M}$, we let $\operatorname{dg} \mathscr{M}$ denote the category of cochain complexes of objects in $\mathscr{M}$. For any two complexes $M$ and $N$ in $\operatorname{dg} \mathscr{M}$ we always let $\operatorname{Hom}_{\mathscr{M}}(M, N)$ denote the hom complex

$$
\operatorname{Hom}_{\mathscr{M}}(M, N)=\bigoplus_{n \in \mathbb{Z}}\left\{\begin{array}{c}
\text { homogenous degree } n \text { maps } \\
M \rightarrow N \text { in } \mathscr{M}
\end{array}\right\} \text {. }
$$

The differential is the usual one

$$
d_{\mathrm{Hom}_{\mathscr{M}}(M, N)}(f)=d_{N} f-(-1)^{|f|} f d_{M} .
$$

We view a "graded object" in $\mathscr{M}$ as a complex in dg $\mathscr{M}$ with vanishing differential. In particular, the cohomology $H^{\bullet}(M)$ of a complex in dg $\mathscr{M}$ will be seen as an object in $\operatorname{dg} \mathscr{M}$ with vanishing differential.

2.2. Quasitriangular Hopf algebras. We follow, for the most part, the notation of [7]. For a quasitriangular Hopf algebra $H$, with $R$-matrix $R \in$ $H \otimes H$, we take $R=\sum_{j} r_{j} \otimes r^{j}$ and $R_{21}=\sum_{j} r^{j} \otimes r_{j}$. We adopt an Einstein sum notation and write $r_{j} \otimes r^{j}$ and $r^{j} \otimes r_{j}$ for these elements respectively. The braiding on the corresponding category $\mathscr{Z}=H \bmod$ will be given by

$$
c_{M, N}(m \otimes n)=\left(r^{j} \cdot n\right) \otimes\left(r_{j} \cdot m\right) .
$$

The $R$-matrix satisfies a number of relations, including the following:

- $\Delta(h) R_{21}=R_{21} \Delta^{o p}(h)$

- $(i d \otimes S)\left(R_{21}\right)=\left(S^{-1} \otimes i d\right)\left(R_{21}\right)=\left(R_{21}\right)^{-1}$,

- $(\epsilon \otimes i d)\left(R_{21}\right)=(i d \otimes \epsilon)\left(R_{21}\right)=1 \otimes 1$. 
- $r^{j} \otimes\left(r_{j}\right)_{1} \otimes\left(r_{j}\right)_{2}=r^{l} r^{j} \otimes r_{l} \otimes r_{j}$,

- $\left(r^{j}\right)_{1} \otimes\left(r^{j}\right)_{2} \otimes r_{j}=r^{j} \otimes r^{l} \otimes r_{l} r_{j}$.

The latter two relations are the braid relations, and all of the relations can be equated with the fact that $c$, as defined at (2.1), is a braiding on the category $H \bmod$.

2.3. The algebra $A * E$ and $Y D_{E}^{E}$. We recall that the category of (right) Yetter-Drinfeld modules over a Hopf algebra $E$ is the category whose objects are vector spaces $M$ equipped with both a right $E$-action and right $E$ coaction which satisfy the compatibility

$$
(m \cdot w)_{0} \otimes(m \cdot w)_{1}=m_{0} \cdot w_{2} \otimes S\left(w_{1}\right) m_{1} w_{3},
$$

for each $m \in M$ and $w \in E$. Morphisms in $Y D_{E}^{E}$ are maps $M \rightarrow N$ which are simultaneously module and comodule maps. This category is braided monoidal, with braiding

$$
c_{M, N}: M \otimes N \rightarrow N \otimes M, \quad m \otimes n \mapsto n_{0} \otimes\left(m \cdot n_{1}\right) .
$$

When $E$ is finite dimensional, the category $Y D_{E}^{E}$ is equal to the category of modules over a quasitriangular Hopf algebra called the Drinfeld, or quantum, double $D$ of $E$. (Or rather, the double of the opposite algebra of $E$ if we follow [7]). So $Y D_{E}^{E}$ can serve as one of our categories $\mathscr{Z}$.

The Hopf algebra $D$ will contain $E^{o p}$ and $E^{*}$ as Hopf subalgebras, and the restriction of the multiplication map $E^{o p} \otimes E^{*} \rightarrow D$ will be a vector space isomorphism. Here $E^{o p}$ means $E$ with the opposite multiplication, usual comultiplication, and inverted antipode $S_{E^{o p}}=S_{E}^{-1}$. If we take a basis $\left\{r_{j}\right\}_{j}$ of $E$, and dual basis $\left\{r^{j}\right\}_{j}$ of $E^{*}$, then the $R$-matrix in $D$ will be the element $\sum_{j} r_{j} \otimes r^{j}$.

Let $A$ be a $E$-module algebra. We denote the given action of $E$ on $A$ by a superscript, ${ }^{w} a:=w \cdot a$, and denote elements in the smash product $A * E$ by juxtaposition $a w=a * w$. Any such smash product $A * E$ becomes an algebra object in $Y D_{E}^{E}$ under the right adjoint action

$$
(a w) \cdot w^{\prime}:=S\left(w_{1}^{\prime}\right) a w w_{2}^{\prime}=\left(S\left(w_{2}^{\prime}\right) a\right) S\left(w_{1}^{\prime}\right) w w_{3}^{\prime}
$$

and obvious coaction $\rho(a w)=a w_{1} \otimes w_{2}$. Or, if we think about $E^{o p}$ in the Drinfeld double $D$, we have the left action given by

$$
w^{\prime} \cdot(a w)=S_{E}\left(w_{1}^{\prime}\right) a w w_{2}^{\prime}
$$

In the above expressions $a \in A$ and $w, w^{\prime} \in E$.

Throughout this work a smash product $A * E$ we be considered as an algebra object in the braided monoidal category $Y D_{E}^{E}$.

2.4. Inner homs. Given any Hopf algebra $H$, the category $H$ mod admits inner homs, i.e. a functor $\underline{\mathrm{Hom}}: H \bmod ^{o p} \times H \bmod \rightarrow H \bmod$ with an adjunction

$$
\operatorname{Hom}_{H \bmod }(L \otimes M, N) \cong \operatorname{Hom}_{H \bmod }(L, \underline{\operatorname{Hom}}(M, N)) .
$$


As a vector space we will have $\underline{\operatorname{Hom}}(M, N)=\operatorname{Hom}_{k}(M, N)$, and the adjunction (2.4) is the usual $\otimes$-Hom adjunction. The action of $H$ on $\underline{\operatorname{Hom}}(M, N)=$ $\operatorname{Hom}_{k}(M, N)$ will be given by $h \cdot f=\left(m \mapsto h_{1} \cdot f\left(S\left(h_{2}\right) \cdot m\right)\right.$, for any $h \in H$, $f \in \operatorname{Hom}_{k}(M, N), m \in M$. One can check easily the identity

$$
\underline{\operatorname{Hom}}(M, N)^{H}=\operatorname{Hom}_{H \bmod }(M, N) \text {. }
$$

Under the aforementioned action the canonical pairing

$$
\underline{\operatorname{Hom}}(M, N) \otimes M \rightarrow N
$$

becomes $H$-linear. Indeed, this is the unique action so that the pairing is $H$-linear.

\section{BRAided Hochschild COHOMOLOGY AS A $\mathscr{Z}$-VALUED INVARIANT}

Take a braided monoidal category $\mathscr{Z}=H \bmod$ and algebra $B$ in $\mathscr{Z}$. In this section we cover all of the basics for the braided Hochschild cohomology $H_{c}^{\bullet}(B)$. We show that the braided cohomology can be identified with the cohomology of a certain canonical complex, which is a braided analog of the standard Hochschild cochain complex. This complex will be a complex in $\mathscr{Z}$, so that each cohomology group $H_{c}^{i}(B)$ will be seen to be an object in $\mathscr{Z}$.

We then go on to consider a relative version of the braided Hochschild cohomology. The relative cohomology will provide an essential tool in establishing relations between the braided Hochschild cohomology and standard, non-braided, Hochschild cohomology.

We first recall some facts from the original work [1]. Given an algebra $B$ in $\mathscr{Z}$ we let $B \underline{o p}$ denote the braided opposite algebra, which has multiplication $b \cdot_{\text {op }} b^{\prime}:=\left(r^{j} \cdot b^{\prime}\right)\left(r_{j} \cdot b\right)$. Given algebras $B$ and $C$ in $\mathscr{Z}$ we let $B \underline{\otimes} C$ denote the braided tensor algebra. This is the vector space $B \otimes C$ with the multiplication

$$
(b \otimes c)\left(b^{\prime} \otimes c^{\prime}\right)=\left(b\left(r^{j} \cdot b^{\prime}\right)\right) \otimes\left(\left(r_{j} \cdot c\right) c^{\prime}\right) .
$$

We let $B \underline{e}$ denote the enveloping algebra $B \underline{o p} \underline{\otimes} B$. The algebra $B$ becomes a right module over $B \underline{e}$ under the action

$$
B \otimes(B \underline{o p} \underline{\otimes} B) \rightarrow B, \quad a \otimes\left(b \otimes b^{\prime}\right) \mapsto\left(r^{j} \cdot b\right)\left(r_{j} \cdot a\right) b^{\prime} .
$$

That is to say, we follow the obvious sequence

$$
(B) \otimes B \otimes B \stackrel{c_{B, B} \otimes i d}{\longrightarrow} B \otimes(B) \otimes B \stackrel{m^{(2)}}{\longrightarrow} B,
$$

where $m^{(2)}$ is the second iteration of the multiplication on $B$.

Definition 3.1. The braided Hochschild cohomology is defined as the extension group $H_{c}^{\bullet}(B):=\operatorname{Ext}_{\bmod B \underline{e}}^{\bullet}(B, B)$

It is shown in [1] that the standard bar resolution

$$
\cdots \rightarrow B \otimes B^{\otimes 2} \otimes B \rightarrow B \otimes B \otimes B \rightarrow B \otimes B \rightarrow 0
$$


becomes a projective resolution of $B$ over $B^{\underline{e}}$ when we endow each $B \otimes$ $B^{\otimes n} \otimes B$ with the $B^{\underline{e}}$ action

$$
\left(a \otimes x \otimes a^{\prime}\right)\left(b \otimes b^{\prime}\right):=\left(r^{j} \cdot b\right)\left(r_{j} \cdot\left(a \otimes x \otimes a^{\prime}\right)\right) b^{\prime},
$$

where $x \in B^{\otimes n}$ and $b\left(a \otimes x \otimes a^{\prime}\right) b^{\prime}=(b a) \otimes x \otimes\left(a^{\prime} b^{\prime}\right)$. (Here $H$ acts diagonally on the higher tensor powers of $B$.) We denote this complex by $\mathscr{B} a r^{c} B$.

To see that $\mathscr{B} a r^{c} B$ is projective in each degree we note that the $B$-action map restricts to give an isomorphism $B^{\otimes n} \otimes B^{\underline{e}} \rightarrow B \otimes B^{\otimes n} \otimes B$, for each $n$. The inverse to this map is given by

$$
\begin{aligned}
& B \otimes B^{\otimes n} \otimes B \rightarrow B^{\otimes n} \otimes B^{\underline{e}}, \\
& b \otimes x \otimes b^{\prime} \mapsto\left(S\left(r_{j}\right) \cdot x\right) \otimes\left(r^{j} \cdot b\right) \otimes b^{\prime}=\left(r_{j} \cdot x\right) \otimes\left(S^{-1}\left(r^{j}\right) \cdot b\right) \otimes b^{\prime}
\end{aligned}
$$

Recall that the category $\mathscr{Z}=H \bmod$ has inner homs. These objects are denoted $\underline{H o m}$ and are equal to the standard $k$-linear homs $\mathrm{Hom}_{k}$ along with the left $H$-action as described in Section 2.4.

Lemma 3.2. For each $n$ we have an isomorphism

$$
\operatorname{Hom}_{\bmod B \underline{e}}\left(B \otimes B^{\otimes n} \otimes B, B\right) \stackrel{\cong}{\operatorname{Hom}}\left(B^{\otimes n}, B\right)
$$

given by restriction. The inverse of the restriction map is given by

$$
\begin{gathered}
\underline{\operatorname{Hom}}\left(B^{\otimes n}, B\right) \rightarrow \operatorname{Hom}_{\bmod B \underline{e}}\left(B \otimes B^{\otimes n} \otimes B, B\right) \\
f \mapsto\left(b \otimes x \otimes b^{\prime} \mapsto\left(r^{j} \cdot b\right)\left(r_{j} \cdot f\right)(x) b^{\prime}\right) .
\end{gathered}
$$

Proof. First note that restriction does in fact provide an isomorphism between $\operatorname{Hom}_{\bmod B \underline{e}}\left(B \otimes B^{\otimes n} \otimes B, B\right)$ and $\underline{\operatorname{Hom}}\left(B^{\otimes n}, B\right)$, since each $B \otimes B^{\otimes n} \otimes B$ is free over $B^{\underline{e}}$. Now, we have for any $F \in \operatorname{Hom}_{\bmod B \underline{e}}\left(B \otimes B^{\otimes n} \otimes B, B\right)$

$$
\begin{aligned}
F\left(b \otimes x \otimes b^{\prime}\right) & =F\left(1 \otimes S\left(r_{j}\right) \cdot x \otimes 1\right)\left(\left(r^{j} \cdot b\right) \otimes b^{\prime}\right) \\
& =\left(r^{l} r^{j} \cdot b\right)\left(r_{l} \cdot F\left(1 \otimes S\left(r_{j}\right) \cdot x \otimes 1\right)\right) b^{\prime} .
\end{aligned}
$$

The braid relation

$$
(i d \otimes \Delta)\left(R_{21}\right)=r^{l} r^{j} \otimes r_{l} \otimes r_{j}
$$

then implies

$$
\left(r^{l} r^{j} \cdot b\right)\left(r_{l} \cdot F\left(1 \otimes S\left(r_{j}\right) \cdot x \otimes 1\right)\right) b^{\prime}=\left(r^{j} \cdot b\right)\left(\left(r_{j}\right)_{1} \cdot F\left(1 \otimes S\left(\left(r_{j}\right)_{2}\right) \cdot x \otimes 1\right)\right) b^{\prime}
$$

If we let $f=F \mid B^{\otimes n}$ the final expression is equal to $\left(r^{j} \cdot b_{0}\right)\left(r_{j} \cdot f\right)(x) b^{\prime}$. This shows that the preimage of $f$ along the restriction isomorphism, which in this case is $F$, is given by the proposed formula (3.3).

As a result of the lemma we get an explicit isomorphism of graded spaces

$$
\operatorname{Hom}_{\bmod B \underline{e}}\left(\mathscr{B} a r^{c} B, B\right) \stackrel{\cong}{\rightarrow} \bigoplus_{n \geq 0} \underline{\operatorname{Hom}}\left(B^{\otimes n}, B\right) .
$$

This induces a differential on the right hand side under which the two complexes will be canonically isomorphic. 
In the following lemma $d_{\mathscr{B} B}$ denotes the standard differential

$$
d_{\mathscr{B} B}\left(b_{1} \otimes \ldots \otimes b_{l}\right)=\sum_{i}(-1)^{i} b_{1} \otimes \ldots \otimes b_{i} b_{i+1} \otimes \ldots \otimes b_{l} .
$$

on the bar construction

$$
\mathscr{B} B=\cdots \rightarrow B^{\otimes 3} \rightarrow B^{\otimes 2} \rightarrow B \stackrel{0}{\rightarrow} k \rightarrow 0 .
$$

The bar construction and bar resolution are related by the formula $\mathscr{B} a r^{c} B=$ $B \otimes \mathscr{B} B \otimes B$. To be precise, one arrives at the bar resolution by taking a "twisted tensor product" between $B$ and the bar construction so that

$$
d_{\mathscr{B} a r^{c} B}=i d_{B} \otimes d_{\mathscr{B} B} \otimes i d_{B}+\text { extremal termal. }
$$

Proposition 3.3. Let $d_{c}$ denote the differential on $\bigoplus_{n} \underline{\operatorname{Hom}}\left(B^{\otimes n}, B\right)$ induced by the restriction isomorphism (3.4).

(1) $d_{c}$ is given in degree 0 by the formula

$$
-d_{c}(f)(b)=\left(r^{j} \cdot b\right)\left(r_{j} \cdot f\right)(1)-f(1) b
$$

and in higher degree by the formula

$$
\begin{aligned}
& (-1)^{|f|+1} d_{c}(f)\left(b \otimes y \otimes b^{\prime}\right) \\
& =\left(r^{j} \cdot b\right)\left(r_{j} \cdot f\right)\left(y \otimes b^{\prime}\right)+f\left(d_{\mathscr{B} B}\left(b \otimes y \otimes b^{\prime}\right)\right)+(-1)^{n+1} f(b \otimes y) b^{\prime},
\end{aligned}
$$

where $f$ is a homogenous map, $y \in B^{\otimes n-2}$, and $b, b^{\prime} \in B$.

(2) Each differential $d_{c}: \underline{\operatorname{Hom}}\left(B^{\otimes n}, B\right) \rightarrow \underline{\operatorname{Hom}}\left(B^{\otimes n+1}, B\right)$ is a map in $\mathscr{Z}$.

Proof. (1) Let $F$ be the preimage of $f$ along the restriction map. Suppose $|f|=n>0$. According to the previous lemma we have

$$
\begin{aligned}
& (-1)^{|f|+1} d_{c}(f)\left(b \otimes y \otimes b^{\prime}\right)=d(F)\left(1 \otimes b \otimes y \otimes b^{\prime} \otimes 1\right) \\
& =F\left(b \otimes y \otimes b^{\prime} \otimes 1\right)+(-1)^{n+1} F\left(1 \otimes b \otimes y \otimes b^{\prime}\right)+F\left(1 \otimes d_{\mathscr{B} B}\left(b \otimes y \otimes b^{\prime}\right) \otimes 1\right) \\
& =\left(r^{j} \cdot b\right)\left(r_{j} \cdot f\right)\left(y \otimes b^{\prime}\right)+f\left(d_{\mathscr{B} B}\left(b \otimes y \otimes b^{\prime}\right)\right)+(-1)^{n+1} f(b \otimes y) b^{\prime} .
\end{aligned}
$$

The formula in degree 0 is verified similarly.

(2) We note first that, for $f \in \underline{\operatorname{Hom}}\left(B^{\otimes n}, B\right), b \in B, x \in B^{\otimes n}$, the element

$$
\left(r^{j} \cdot b\right)\left(r_{j} \cdot f\right)(x)
$$

is the image of the monomial $f \otimes b \otimes x$ under the sequence of maps

$\underline{\operatorname{Hom}}\left(B^{\otimes n}, B\right) \otimes B \otimes B^{\otimes n} \stackrel{c \otimes i d}{\longrightarrow} B \otimes \underline{\operatorname{Hom}}\left(B^{\otimes n}, B\right) \otimes B^{\otimes n} \stackrel{i d \otimes \text { pair }}{\longrightarrow} B \otimes B \stackrel{\text { mult }}{\longrightarrow} B$, all of which are morphisms in $\mathscr{Z}=H \bmod$. So for any $h \in H$ we have

$$
h \cdot\left(\left(r^{j} \cdot b\right)\left(r_{j} \cdot f\right)(x)\right)=\left(r^{j} h_{2} \cdot b\right)\left(r_{j} h_{1} \cdot f\right)\left(h_{3} \cdot x\right) .
$$

Replacing $h \otimes b \otimes x$ with $h_{1} \otimes\left(S\left(h_{3}\right) \cdot b\right) \otimes\left(S\left(h_{2}\right) \cdot x\right)$ then gives

$$
\begin{aligned}
& h_{1} \cdot\left(\left(r^{j} S\left(h_{3}\right) \cdot b\right)\left(r_{j} \cdot f\right)\left(S\left(h_{2}\right) \cdot x\right)\right) \\
& =\left(r^{j} h_{2} S\left(h_{5}\right) \cdot b\right)\left(r_{j} h_{1} \cdot f\right)\left(h_{3} S\left(h_{4}\right) \cdot x\right) \\
& =\left(r^{j} h_{2} \epsilon\left(h_{3}\right) S\left(h_{4}\right) \cdot b\right)\left(r_{j} h_{1} \cdot f\right)(\cdot x) \\
& =\left(r^{j} \cdot b\right)\left(r_{j} h \cdot f\right)(x) .
\end{aligned}
$$


One can also check the more obvious relations

$$
h_{1} \cdot\left(f\left(d_{\mathscr{B} B}\left(S\left(h_{2}\right) \cdot x\right)\right)\right)=h_{1} \cdot\left(f\left(S\left(h_{2}\right) \cdot d_{\mathscr{B} B}(x)\right)\right)=(h \cdot f)\left(d_{\mathscr{B} B}(x)\right)
$$

and

$$
h_{1} \cdot\left(f\left(S\left(h_{3}\right) \cdot x\right)\left(S\left(h_{2}\right) \cdot b^{\prime}\right)\right)=\left(h_{1} \cdot f\left(S\left(h_{2}\right) \cdot x\right)\right) b^{\prime}=(h \cdot f)(x) b^{\prime} .
$$

So we see that for $x=b \otimes y \otimes b^{\prime}$ we have

$$
\begin{aligned}
& (-1)^{|f|+1}\left(h \cdot d_{c}(f)-d_{c}(h \cdot f)\right)(x) \\
& =(-1)^{|f|+1}\left(\left(h \cdot d_{c}(f)\right)\left(b \otimes y \otimes b^{\prime}\right)-d_{c}(h \cdot f)\left(b \otimes y \otimes b^{\prime}\right)\right) \\
& =h_{1} \cdot\left(\left(r^{j} S\left(h_{3}\right) \cdot b\right)\left(r_{j} \cdot f\right)\left(S\left(h_{2}\right) \cdot\left(y \otimes b^{\prime}\right)\right)\right)-\left(r^{j} \cdot b\right)\left(r_{l} h \cdot f\right)\left(y \otimes b^{\prime}\right) \\
& +h_{1} \cdot\left(f\left(d_{\mathscr{B} B}\left(S\left(h_{2}\right) x\right)\right)\right)-(h \cdot f)\left(d_{\mathscr{B} B}(x)\right) \\
& +h_{1} \cdot\left(f\left(S\left(h_{3}\right) \cdot(b \otimes y)\right)\left(S\left(h_{2}\right) \cdot b^{\prime}\right)\right)-(h \cdot f)(b \otimes y) b^{\prime} .
\end{aligned}
$$

Checking the above expressions (3.6)-(3.8), we see that the final sum is 0 . So $h \cdot d_{c}(f)=d_{c}(h \cdot f)$ and therefore $d_{c}$ is $H$-linear .

Definition 3.4. We let $C_{c}^{\bullet}(B)$ denote the complex

$$
C_{c}^{\bullet}(B)=0 \rightarrow \underline{\operatorname{Hom}}(k, B) \stackrel{d_{c}^{0}}{\operatorname{Hom}}(B, B) \stackrel{d_{c}^{1}}{\rightarrow} \underline{\operatorname{Hom}}\left(B^{\otimes 2}, B\right) \rightarrow \cdots,
$$

where $d_{c}$ is given by the formula (3.5). We call this complex the braided Hochschild cochain complex.

Proposition 3.3 tells us that $C_{c}^{\bullet}(B)$ is a chain complex in $\mathscr{Z}$. So we get

Theorem 3.5. (1) The braided Hochschild cohomology $H_{c}^{\bullet}(B)$ is the cohomology of the braided Hochschild complex $C_{c}^{\bullet}(B)$.

(2) The cohomology $H_{c}^{\bullet}(B)$ is an object in $\operatorname{dg} \mathscr{Z}$ (with vanishing differential).

Proof. Statement (1) follows from Lemma 3.2 and the construction of the differential $d_{c}$. Statment (2) follows from (1) and Proposition 3.3.

Remark 3.6. The complex $C_{c}^{\bullet}(B)$ may be legitimate even when we replace $\mathscr{Z}$ with an arbitrary braided multi-tensor category, with possibly nontrivial associator. This is despite the fact that Definition 3.1 may make no sense in this setting.

3.1. Relative braided cohomology. Given any subalgebra $E \subset B$ in $\mathscr{Z}$ we can define the relative bar resolution $\mathscr{B} a r_{E}^{c} B$ to be the complex

$$
\cdots \rightarrow B \otimes_{E} B^{\otimes_{E} 2} \otimes_{E} B \rightarrow B \otimes_{E} B \otimes_{E} B \rightarrow B \otimes_{E} B \rightarrow 0
$$

with the usual differential and right $B$-action given by the same formula (3.1). We define the E-relative Hochschild cohomology as the cohomology

$$
H_{c, E}^{\bullet}(B)=H^{\bullet}\left(\operatorname{Hom}_{\bmod B \underline{e}}\left(\mathscr{B} a r_{E}^{c} B, B\right)\right) .
$$


We similarly let $\mathscr{B}_{E} B$ denote the relative bar construction

$$
\mathscr{B}_{E} B=\cdots \rightarrow B^{\otimes_{E} 3} \rightarrow B \otimes_{E} B \rightarrow B \stackrel{0}{\rightarrow} E \rightarrow 0
$$

with the usual differential, as above.

We have an embedding

$$
\operatorname{Hom}_{\bmod B \underline{e}}\left(\mathscr{B} a r_{E}^{c} B, B\right) \rightarrow \operatorname{Hom}_{\bmod B \underline{e}}\left(\mathscr{B} a r^{c} B, B\right)=C_{c}^{\bullet}(B)
$$

of cochain complexes dual to the projection $\mathscr{B} a r^{c} B \rightarrow \mathscr{B} a r_{E}^{c} B$. In order to think clearly about the image of this embedding we introduce a new category.

Definition 3.7. Let $E$ be an algebra in $\mathscr{Z}$. We let ${ }_{E} \mathscr{Z}_{E}$ denote the category of objects $M$ in $\mathscr{Z}$ with an additional $E$-bimodule structure such that the action $E \otimes M \otimes E \rightarrow M$ is a map in $\mathscr{Z}$. Morphisms in this category will be maps in $\mathscr{Z}$ which are also E-bimodule maps.

Both $B^{\otimes n}$ and $B^{\otimes_{E} n}$ will be objects in ${ }_{E} \mathscr{Z}_{E}$, for example. The projections $B^{\otimes n} \rightarrow B^{\otimes_{E} n}$ will be maps in ${ }_{E} \mathscr{Z}_{E}$.

The category ${ }_{E} \mathscr{Z}_{E}$ is actually the category of modules over a certain smash-product-esque algebra [15, Lemma 3.2]. Whence ${ }_{E} \mathscr{Z}_{E}$ will be abelian with enough projectives. There is a natural functor

$$
\text { Forget }:{ }_{E} \mathscr{Z}_{E} \rightarrow \bmod E^{\underline{e}}
$$

which sends an object $M$ to the vector space $M$ with the right $E$-action $x \cdot\left(w \otimes w^{\prime}\right)=\left(r^{j} \cdot w\right)\left(r_{j} \cdot x\right) w^{\prime}$ for each $x \in M, w, w^{\prime} \in E$.

Given any $M$ and $N$ in $E_{E} \mathscr{Z}_{E}$ the maps in $\operatorname{Hom}_{\bmod E e}(M, N)$ are exactly those $k$-linear maps $f$ which satisfy the additional relations

$$
f(x w)=f(x) w
$$

and

$$
f(w x)=\left(r^{j} \cdot w\right)\left(r_{j} \cdot f\right)(x)
$$

for each $x \in M, w \in E$. The relation (3.11) is obviously just right $E$-linearity while (3.12) should be familiar from Lemma 3.2.

Recall that the set of inner homs $\underline{\operatorname{Hom}}(M, N)$ in $\mathscr{Z}$ is equal to the set of $k$-linear maps, after we forget the $\mathscr{Z}$-structure.

Lemma 3.8. In each degree $n$, the image of each embedding (3.9) is exactly the subset

$$
\operatorname{Hom}_{\bmod E \underline{e}}\left(B^{\otimes_{E} n}, B\right) \subset \underline{\operatorname{Hom}}\left(B^{\otimes n}, B\right) .
$$

It may actually be more informative to think instead about the sequence of inclusions

$$
\operatorname{Hom}_{\bmod E \underline{e}}\left(B^{\otimes_{E} n}, B\right) \subset \underline{\operatorname{Hom}}\left(B^{\otimes_{E} n}, B\right) \subset \underline{\operatorname{Hom}}\left(B^{\otimes n}, B\right)
$$

Proof. This follows from the formula for the identification (3.3) of Lemma

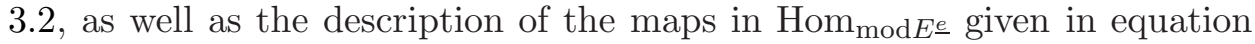
(3.11) and (3.12). 
Lemma 3.9. For any $M, N$ in ${ }_{E} \mathscr{Z}_{E}$, the embedding $\operatorname{Hom}_{\bmod E e}(M, N) \rightarrow$ $\underline{\operatorname{Hom}}(M, N)$ realizes $\operatorname{Hom}_{\bmod E \underline{e}}(M, N)$ as an object in $\mathscr{Z}$.

Proof. We need to show that each $\operatorname{Hom}_{\bmod E \underline{e}}(M, N)$ is stable under the action of $H$, where $\mathscr{Z}=H$ mod. A straightforward computation shows that the subspace of right $E$-linear maps in a $H$-submodule in $\underline{\operatorname{Hom}}(M, N)$, so we need only concern ourselves with the relation (3.12).

Suppose $f \in \underline{\operatorname{Hom}}(M, N)$ satisfies the relation (3.12) and take any $h \in H$. Then

$$
\left.(h \cdot f)(w x)=h_{1} \cdot\left(\left(r^{j} S\left(h_{3}\right) \cdot w\right)\left(r_{j} \cdot f\right)\left(S\left(h_{2}\right) \cdot x\right)\right)\right) .
$$

We find just as in the proof of Proposition 3.3 (2) the equality

$$
\left.h_{1} \cdot\left(\left(r^{j} S\left(h_{3}\right) \cdot w\right)\left(r_{j} \cdot f\right)\left(S\left(h_{2}\right) \cdot x\right)\right)\right)=\left(r^{j} \cdot w\right)\left(r_{j} h \cdot f\right)(x),
$$

which gives the desired relation

$$
(h \cdot f)(w x)=\left(r^{j} \cdot w\right)\left(r_{j} h \cdot f\right)(x) .
$$

So we see that $\operatorname{Hom}_{\bmod E \underline{e}}(M, N) \subset \underline{\operatorname{Hom}}(M, N)$ is an $H$-submodule.

In order to emphasize the natural $\mathscr{Z}$-structure on these hom sets we adopt

Notation 3.10. For any $M$ and $N$ in $E_{E} \mathscr{Z}_{E}$ we let $\underline{\operatorname{Hom}}_{E}(M, N)$ denote the set of maps $\operatorname{Hom}_{\bmod E \underline{e}}(M, N)$ along with its $\mathscr{Z}$-structure induced by the inclusion $\operatorname{Hom}_{\bmod E \underline{e}}(M, N) \rightarrow \underline{\operatorname{Hom}}(M, N)$.

We remark that this hom set may not truly be an inner hom set in a category, despite the fact that we have elected to adopt the underline notation.

Lemma 3.9 implies that the image of $\operatorname{Hom}_{\bmod B \underline{e}}\left(\mathscr{B} a r_{E}^{c} B, B\right)$ in $C_{c}^{\bullet}(B)$ will be a subcomplex in $\mathscr{Z}$. So the complex $\operatorname{Hom}_{\bmod B e}\left(\mathscr{B} a r_{E}^{c} B, B\right)$ becomes naturally an object in $\operatorname{dg} \mathscr{Z}$, and the relative braided Hochschild cohomology will again be $\mathscr{Z}$-valued.

We now write down an explicit complex $C_{c, E}^{\bullet}(B)$ analogous to the braided Hochschild cochain complex constructed in the non-relative instance: first, Lemma 3.8 tells us that the image of the embedding (3.9) is the graded subspace

$$
\bigoplus_{n \in \mathbb{Z}} \underline{\operatorname{Hom}}_{E}\left(B^{\otimes_{E} n}, B\right) \subset C_{c}^{\bullet}(B) .
$$

Second, since (3.9) is a chain map we see that this subspace is a subcomplex under the differential on $C_{c}^{\bullet}(B)$. So restricting to the generators now provides a cochain complex isomorphism

$$
\operatorname{Hom}_{\bmod B \underline{e}}\left(\mathscr{B} a r_{E}^{c} B, B\right) \stackrel{\cong}{\rightarrow}\left(\bigoplus_{n \in \mathbb{Z}} \underline{\operatorname{Hom}}_{E}\left(B^{\otimes_{E} n}, B\right), d_{c}\right),
$$

where the differential $d_{c}$ is given by the formula (3.5).

Definition 3.11. We let $C_{c, E}^{\bullet}(B)$ denote the complex

$$
C_{c, E}^{\bullet}(B)=0 \rightarrow \underline{\operatorname{Hom}}_{E}(E, B) \stackrel{d_{c}^{0}}{\rightarrow} \underline{\operatorname{Hom}}_{E}(B, B) \stackrel{d_{c}^{1}}{\rightarrow} \underline{\operatorname{Hom}}_{E}\left(B^{\otimes_{E}} 2, B\right) \stackrel{d_{c}^{2}}{\rightarrow} \cdots .
$$


The following proposition is now clear.

Proposition 3.12. We have $H_{c, E}^{\bullet}(B)=H^{\bullet}\left(C_{c, E}^{\bullet}(B)\right)$, and the cohomology $H_{c, E}^{\bullet}(B)$ is an object in $\operatorname{dg} \mathscr{Z}$.

Our main intent in introducing the relative cohomology is to provide a relationship between the braided Hochschild cohomology and standard Hochschild cohomology. With the above information we will be able to relate the standard Hochschild cohomology to the relative braided cohomology. (This is done in Section 4.) We would like to establish finally some conditions under which the relative and non-relative braided Hochschild cohomologies agree.

Definition 3.13. We say $E$ is separable in $\mathscr{Z}$ if the category ${ }_{E} \mathscr{Z}_{E}$ is semisimple.

Lemma 3.14. (1) For any $M$ and $N$ in ${ }_{E} \mathscr{Z}_{E}$ there is an equality

$$
\operatorname{Hom}_{E} \mathscr{Z}_{E}(M, N)=\underline{\operatorname{Hom}}_{E}(M, N)^{\mathscr{Z}} \text {. }
$$

(2) If $\mathscr{Z}$ is semisimple, then $E$ is separable in $\mathscr{Z}$ whenever $E$ e is semisimple as an ordinary $k$-algebra.

Note that both $\operatorname{Hom}_{E} \mathscr{Z}_{E}(M, N)$ and $\underline{\operatorname{Hom}}_{E}(M, N)$ are subsets in $\operatorname{Hom}_{k}(M, N)$. So it makes sense to propose that the two sets are equal.

Proof. (1) Maps in both hom sets are right E-linear, as well as maps in $\mathscr{Z}$. So we need only concern ourselves with left $E$-linearity. Any $H$-invariant (i.e. $H$-linear) function $f$ in $\underline{\operatorname{Hom}}_{E}(M, N)$ will satisfy $h \cdot f=\epsilon(h) f$ for each $h \in H$. Therefore, for any $w \in E, m \in M$, we have

$$
f(w m)=\left(r^{j} \cdot w\right)\left(r_{j} \cdot f\right)(m)=\left(r^{j} \epsilon\left(r_{j}\right) \cdot w\right) f(m)=w f(m) .
$$

Whence $f$ is left $E$-linear. So $\underline{\operatorname{Hom}}_{E}(M, N)^{\mathscr{Z}} \subset \operatorname{Hom}_{E} \mathscr{Z}_{E}(M, N)$.

Recall now that maps in ${ }_{E} \mathscr{Z}_{E}$ are $H$-linear, and hence $H$-invariant. Therefore, for any $g \in \operatorname{Hom}_{E} \mathscr{Z}_{E}(M, N)$,

$$
g(w m)=w g(m)=\left(r^{j} \cdot w\right)\left(r_{j} \cdot g\right)(m) .
$$

So we get the opposite inclusion as well, and hence an equality.

(2) Note that $\mathscr{Z}$ is semisimple if and only if the invariants functor $(-)^{\mathscr{Z}}$ is exact. So, if $\mathscr{Z}$ and $E$ e are semisimple then for any $M$ in ${ }_{E} \mathscr{Z}_{E}$ the hom functor $\operatorname{Hom}_{E} \mathscr{Z}_{E}(M,-)$ is exact, since it will be the composite of the exact functors

$$
(-)^{\mathscr{Z}} \circ \underline{\operatorname{Hom}}_{E}(M,-)=(-)^{\mathscr{Z}} \circ \operatorname{Hom}_{\bmod E \underline{e}}(M,-)
$$

by (1). So all objects in $\mathscr{Z}_{E}$ are projective, and $E$ is separable in $\mathscr{Z}$.

The following is a braided version of a result of Gerstenhaber and Schack [10].

Proposition 3.15. If $E$ is separable in $\mathscr{Z}$ then

(1) the inclusion $C_{c, E}^{\bullet}(B) \rightarrow C_{c}^{\bullet}(B)$ is a quasi-isomorphism,

(2) there induced map $H_{c, E}^{\bullet}(B) \rightarrow H_{c}^{\bullet}(B)$ is an isomorphism in $\operatorname{dg} \mathscr{Z}$. 
Proof. The second statement follows from the first. So we address (1). First, note that for any $M$ in ${ }_{E} \mathscr{Z}_{E}, B \otimes M \otimes B$ and $B \otimes_{E} M \otimes_{E} B$ become objects in $\bmod B^{\underline{e}}$ under the obvious action(s)

$$
\left(a \otimes m \otimes a^{\prime}\right) *\left(b \otimes b^{\prime}\right)=\left(r^{j} \cdot b\right)\left(r_{j} \cdot\left(a \otimes m \otimes a^{\prime}\right)\right) b^{\prime} .
$$

As was the case above, the braiding gives an isomorphism between each $B \otimes M \otimes B$ and the free $B \underline{e}$ module $M \otimes B$ e.

Now, If $E$ is separable in $\mathscr{Z}$ then for any $M$ in ${ }_{E} \mathscr{Z}_{E}$ the action map $m_{M}: E \otimes M \otimes E \rightarrow M$ will admit a splitting $\sigma_{M}: M \rightarrow E \otimes M \otimes E$ in ${ }_{E} \mathscr{Z}_{E}$. The multiplication map $m: E \otimes E \rightarrow E$ will also be split in ${ }_{E} \mathscr{Z}_{E}$ by some $\sigma_{E}: E \rightarrow E \otimes E$. Whence the projections

$$
B \otimes M \otimes B=B \otimes_{E} E \otimes M \otimes E \otimes_{E} B \stackrel{i d \otimes m_{M} \otimes i d}{\rightarrow} B \otimes_{E} M \otimes_{E} B
$$

and

$$
B \otimes B=B \otimes_{E} E \otimes E \otimes_{E} B \stackrel{i d \otimes m \otimes i d}{\rightarrow} B \otimes_{E} B
$$

will be split in $\bmod B^{\underline{e}}$ by $1 \otimes \sigma_{M} \otimes 1$ and $1 \otimes \sigma_{E} \otimes 1$ respectively. So $B \otimes_{E} M \otimes_{E} B$ and $B \otimes_{E} B$ are summands of free modules and hence projective in $\bmod B^{e}$.

By the above general argument we find that each $B \otimes_{E} B^{\otimes_{E} n} \otimes_{E} B$ is projective and $\mathscr{B} a r_{E}^{c} B \rightarrow B$ is a projective resolution of $B$ in $\bmod B^{e}$. Consequently the projection $\mathscr{B} a r^{c} B \rightarrow \mathscr{B} a r_{E}^{c} B$ is a homotopy equivalence, and applying the functor $\operatorname{Hom}_{\bmod B} \underline{e}(-, B)$ produces a quasi-isomorphism

$$
\operatorname{Hom}_{\bmod B \underline{e}}\left(\mathscr{B} a r_{E}^{c} B, B\right) \stackrel{\sim}{\rightarrow} \operatorname{Hom}_{\bmod B \underline{e}}\left(\mathscr{B} a r^{c} B, B\right) \text {. }
$$

Under the identifications

$\operatorname{Hom}_{\bmod B \underline{e}}\left(\mathscr{B} a r_{E}^{c} B, B\right)=C_{c, E}^{\bullet}(B)$ and $\operatorname{Hom}_{\bmod B \underline{e}}\left(\mathscr{B} a r^{c} B, B\right)=C_{c, E}^{\bullet}(B)$, which are both given by restriction, (3.13) becomes the canonical embedding $C_{c, E}^{\bullet}(B) \rightarrow C_{c}^{\bullet}(B)$. We see now that the embedding is a quasi-isomorphism.

\section{IDENTIFICATIONS With STANDARD HochSCHILD COHOMOLOGY FOR SMASH PRODUCTS}

We fix $E$ to be a finite dimensional Hopf algebra, $A$ an $E$-module algebra, $\mathscr{Z}=Y D_{E}^{E}$, and $B=A * E$. We take $A * E$ to be an algebra in $Y D_{E}^{E}$ as described in Section 2.3. We can recover $A$ in a functorial manner from $B$ as the coinvariants $A=B^{c o E}$. The subalgebra $E \subset B$ becomes a subalgebra in $Y D_{E}^{E}$ under the adjoint action of $E$ on itself and regular right coaction. So we may consider the relative cohomology $H_{c, E}^{\bullet}(B)$.

Recall from the relation (3.11) that any map in $\underline{\operatorname{Hom}}_{E}\left(B^{\otimes_{E} n}, B\right)$ will be right $E$-linear.

Lemma 4.1. When $B=A * E$, then the inclusion

$$
\underline{\operatorname{Hom}}_{E}\left(B^{\otimes_{E} n}, B\right) \subset \operatorname{Hom}_{\bmod E}\left(B^{\otimes_{E} n}, B\right)
$$

is an equality. 
Proof. We let juxtaposition denote the multiplication in $B$, and the dot denote the action of $E^{o p}$ according to the Yetter-Drinfeld structure. Take $D$ to be the double of $E$ so that $Y D_{E}^{E}=D \bmod$. We verify that for any right $E$-linear $f: B^{\otimes_{E} n} \rightarrow B$ the equation (3.12) will already be satisfied.

Note that, since each tensor product is over $E$, we will have for any $w \in E^{o p} \subset D$ identities

$$
\begin{aligned}
w \cdot\left(b_{1} \otimes \ldots \otimes b_{l}\right) & =\left(S_{E}\left(w_{1}\right) b_{1} w_{2}\right) \otimes\left(S_{E}\left(w_{3}\right) b_{2} w_{4}\right) \otimes \ldots \otimes\left(S_{E}\left(w_{2 l-1}\right) b_{l} w_{2 l}\right) \\
& =\left(S_{E}\left(w_{1}\right) b_{1}\right) \otimes b_{2} \otimes \ldots \otimes\left(b_{l} w_{2}\right)
\end{aligned}
$$

and hence, for any $w \in E^{o p} \subset D$ and $x \in B^{\otimes_{E} n}$,

$$
w x=\left(S_{E}^{-1}\left(w_{2}\right) \cdot x\right) w_{1} .
$$

It follows that for any right $E$-linear $f: B^{\otimes_{E} n} \rightarrow B$ we have

$$
\begin{array}{rlr}
f(w x) & =f\left(\left(S_{E}^{-1}\left(w_{2}\right) \cdot x\right) w_{1}\right) & \\
& =f\left(S_{E}^{-1}\left(w_{2}\right) \cdot x\right) w_{1} & \text { by right E-linearity } \\
& =w_{1} S_{E}\left(w_{2}\right)\left(f\left(S_{E}^{-1}\left(w_{4}\right) \cdot x\right) w_{3}\right) & \\
& =w_{1}\left(S_{E}\left(w_{2}\right) f\left(S_{E}^{-1}\left(w_{4}\right) \cdot x\right) w_{3}\right) \\
& =w_{1}\left(w_{2} \cdot\left(f\left(S_{E}^{-1}\left(w_{3}\right) \cdot x\right)\right)\right) \\
& =w_{1}\left(w_{2} \cdot f\right)(x)
\end{array}
$$

Now if we view $E$ as a subcomodule in $B$ then $w_{1} \otimes w_{2}=w_{0} \otimes w_{1}=r^{j} \cdot w \otimes r_{j}$ and the final equation above becomes

$$
f(w x)=\left(r^{j} \cdot w\right)\left(r_{j} \cdot f\right)(x) .
$$

Whence (3.12) is satisfied.

By the lemma, when $B=A * E$, the complex $C_{c, E}^{\bullet}(B)$ now appears as

$$
\begin{aligned}
& C_{c, E}^{\bullet}(B) \\
& =0 \rightarrow \operatorname{Hom}_{\bmod E}(E, B) \stackrel{d_{c}^{0}}{\rightarrow} \operatorname{Hom}_{\bmod E}(B, B) \stackrel{d_{c}^{1}}{\rightarrow} \operatorname{Hom}_{\bmod E}\left(B^{\otimes_{E}}, B\right) \stackrel{d_{c}^{2}}{\rightarrow} \cdots .
\end{aligned}
$$

The complex $C_{c, E}^{\bullet}(B)$ in this case is exactly the "intermediate complex" of $\left[24\right.$, Ch. 3]. ${ }^{1}$

In the following proposition the notation $C^{\bullet}(A, B)$ denotes the standard, i.e. non-braided, Hochschild cochain complex of $A$ with coefficients in $B$. Note that restricting along the embeddings $A^{\otimes n} \rightarrow B^{\otimes_{E} n}$ produces vector space maps

$$
C_{c, E}^{n}(B)=\underline{\operatorname{Hom}}_{E}\left(B^{\otimes_{E} n}, B\right) \rightarrow \operatorname{Hom}_{k}\left(A^{\otimes n}, B\right)=C^{n}(A, B)
$$

in each degree $n$.

Proposition 4.2. Restriction provides an isomorphism of chain complexes $C_{c, E}^{\bullet}(B) \stackrel{\cong}{\rightarrow} C^{\bullet}(A, B)$ and subsequent isomorphism of cohomologies $H_{c, E}^{\bullet}(B) \stackrel{\cong}{\rightarrow}$ $H H^{\bullet}(A, B)$.

\footnotetext{
${ }^{1}$ At the point of $[24]$ the relation with braided cohomology was still obscured.
} 
Proof. The fact that the restriction maps are isomorphisms follows immediately from the fact that the inclusion $A^{\otimes n} \otimes E=A^{\otimes(n-1)} \otimes B \rightarrow B^{\otimes_{E} n}$ is an isomorphism of right $E$-modules. As for the differential we note that, by coinvariance of elements in $A$ and the formula for the differential $d_{c}$ given at (3.5), we have

$$
\begin{aligned}
& (-1)^{n+1} d_{c}(f)\left(a_{1} \otimes \ldots \otimes a_{n+1}\right) \\
& =a_{1} f\left(a_{2} \otimes \ldots \otimes a_{n+1}\right)+(-1)^{n+1} f\left(a_{1} \otimes \ldots a_{n}\right) a_{n+1} \\
& +\sum_{i=1}^{n}(-1)^{i} f\left(a_{1} \otimes \ldots a_{i} a_{i+1} \otimes \ldots \otimes a_{n+1}\right)
\end{aligned}
$$

for any degree $n$ function $f \in C_{c}^{n}(B)$ and $a_{i} \in A \subset B$. This is exactly the non-braided Hochschild differential.

As a consequence of the above isomorphism, and the fact that $C_{c, E}^{\bullet}(B)$ is a complex in $Y D_{E}^{E}$, we see that the Hochschild complex $C^{\bullet}(A, B)$ is a complex in $Y D_{E}^{E}$. We see also that the cohomology is a graded object in $Y D_{E}^{E}$.

One can check easily that the right $E$-action implicit in this YetterDrinfeld structure is exactly the right action considered in [32, 25]. The coaction is induced by the coaction on $B=A * E$. Namely, by coinvariance of the elements in $A$, we will have for any $f \in C^{\bullet}(A, B), \xi \in E^{*}$, and $x \in \mathscr{B} A$

$$
(\xi \cdot f)(x)=\xi_{1} \cdot\left(f\left(S\left(\xi_{2}\right) \cdot x\right)\right)=\xi \cdot(f(x)) .
$$

Written another way, the coproduct $f_{0} \otimes f_{1}$ of $f$ is the unique element so that

$$
f_{0}(x) \otimes f_{1}=(f(x))_{0} \otimes(f(x))_{1}
$$

for each $x \in \mathscr{B} A$. In the case of a finite group $\operatorname{ring} G$, for example, the $G$ coaction on $C^{\bullet}(A, B)=C^{\bullet}(A, A * G)$ corresponds to the obvious $G$-grading

$$
C^{\bullet}(A, A * G)=C^{\bullet}\left(A, \bigoplus_{g \in G} A g\right)=\bigoplus_{g \in G} C^{\bullet}(A, A g) .
$$

We now want to address the question of when $H^{\bullet}(A, B)$ is equal to the non-relative braided Hochschild cohomology. In light of Proposition 3.15, it suffices to understand when $E$ is separable in $Y D_{E}^{E}$. We will see that this occurs when $E$ is both semisimple and cosemisimple.

Lemma 4.3. The vector space equality $E=E \underline{o p}$ is an equality of algebras in $Y D_{E}^{E}$.

Proof. The equality is already an identification in $Y D_{E}^{E}$, so we need only deal with the multiplication. We denote the adjoint action of $E^{o p} \subset D$ by a dot $\cdot$, and multiplication by juxtaposition. For any $w, w^{\prime} \in E$,

$$
w \cdot \underline{o p} w^{\prime}=\left(w_{1}^{\prime}\right)\left(w_{2}^{\prime} \cdot w\right)=\left(w_{1}^{\prime}\right)\left(S\left(w_{2}^{\prime}\right) w w_{3}^{\prime}\right)=\left(w_{1}^{\prime} S\left(w_{2}^{\prime}\right)\right) w w_{3}^{\prime}=w w^{\prime},
$$

and we are done.

Lemma 4.4. If $E$ is semisimple and cosemisimple then the algebra $E^{\underline{e}}$ is separable in $Y D_{E}^{E}$. 
Proof. Since $E$ is both semisimple and cosemisimple the double $D$ is semisimple (see e.g. [21]). Hence $Y D_{E}^{E}=D \bmod$ is semisimple. It therefore suffices to establish semisimplicity of the braided enveloping algebra $E^{e}$, by Lemma 3.14. By the previous lemma we have $E^{\underline{e}}=E \underline{\otimes}$. Let $E_{1}$ and $E_{2}$ denote the left and right copies of $E$ in the product $E \underline{\otimes E}$. Then, by checking the multiplication

$$
(a \otimes b)\left(a^{\prime} \otimes b^{\prime}\right)=a\left(a_{1}^{\prime}\right) \otimes\left(a_{2}^{\prime} \cdot b\right) b^{\prime}=a\left(a_{1}^{\prime}\right) \otimes\left(S\left(a_{2}^{\prime}\right) b a_{3}^{\prime}\right) b^{\prime}
$$

we see that $E_{1} \underline{\otimes} E_{2}$ is the smash product $E_{1} * E_{2}$, where $E_{2}$ is a right $E_{1}$ module algebra under the adjoint action. Now we have the standard identification of maps over the smash product as the invariants

$$
\operatorname{Hom}_{\bmod E \underline{e}}(-,-)=\operatorname{Hom}_{\bmod E_{1} * E_{2}}(-,-)=\operatorname{Hom}_{\bmod E_{2}}(-,-)^{E_{1}} \text {. }
$$

Semisimplicity of $E=E_{1}=E_{2}$ then implies exactness of the functor

$$
\operatorname{Hom}_{\bmod E \underline{e}}(M,-)=\operatorname{Hom}_{\bmod E_{2}}(M,-)^{E_{1}}
$$

for arbitrary $M$ in $\bmod E^{e}$. So all objects in $\bmod E^{e}$ are projective, and $E^{e}$ is semisimple.

Lemma 4.4 in conjunction with Proposition 3.15 then gives

Theorem 4.5. Suppose that $E$ is semisimple and cosemisimple, and $B=$ $A * E$. Then there is a canonical isomorphism between the E-relative braided cohomology and non-relative braided cohomology $H_{c, E}^{\bullet}(B) \cong H_{c}^{\bullet}(B)$ in $\operatorname{dg} Y D_{E}^{E}$.

The following Corollary was proved in the unpublished work of Schedler and Witherspoon in the case of a finite group $E=k G$ in characteristic 0 [28]. The methods employed in [28] were quite different.

Corollary 4.6. (Generalized Schedler-Witherspoon Isomorphism) When E is semisimple and cosemisimple, and $B=A * E$, there is a canonical isomorphism $H H^{\bullet}(A, B) \cong H_{c}^{\bullet}(B)$.

Proof. By Proposition 4.2 and Theorem 4.5 we have $H H^{\bullet}(A, B) \cong H_{c, E}^{\bullet}(B) \cong$ $H_{c}^{\bullet}(B)$.

As noted above, the Yetter-Drinfeld structure on $H H^{\bullet}(A, B)$ induced by this isomorphism is the expected one, with the action from [32, 25] and coaction induced from $B=A * E$. As a consequence of the above results and Ştefan's spectral sequence we also have

Corollary 4.7. When $E$ is semisimple and cosemisimple there is a vector space identification $H H^{\bullet}(B)=H_{c}^{\bullet}(B)^{E}$ and for general $E$ there is a spectral sequence

$$
\operatorname{Ext}_{\bmod E}\left(k, H_{c, E}^{\bullet}(B)\right) \Rightarrow H H^{\bullet}(B)
$$

Proof. Ştefan's spectral sequence [32] appears as

$$
\operatorname{Ext}_{\bmod E}\left(k, H H^{\bullet}(A, B)\right) \Rightarrow H H^{\bullet}(B) .
$$

So the result follows from Proposition 4.2 and Theorem 4.5. 
4.1. An application to deformation theory. Take $B=A * E$. For this subsection we would like to employ the normalized complex $\bar{C}_{c, E}^{\bullet}(B)$. This consists of all maps in the complex $C_{c, E}^{\bullet}(B)$ which vanish on any monomial $b_{1} \otimes \ldots \otimes b_{n}$ with $b_{i}=1$ for some $i$. The inclusion $\bar{C}_{c, E}^{\bullet}(B) \rightarrow C_{c, E}^{\bullet}(B)$ will be a quasi-isomorphism, for the same reason as it is the case in the non-braided setting $[11,10]$. Namely, the kernel of the projection $\mathscr{B} a r_{E}^{c} B \rightarrow \mathscr{B}^{-} r_{E}^{c} B$, to which the embedding $\bar{C}_{c, E}^{\bullet}(B) \rightarrow C_{c, E}^{\bullet}(B)$ is dual, will (still) be contractible.

In the following we take $L(B)$ to be the shifted, normalized, non-braided Hochschild complex $\Sigma \bar{C}^{\bullet}(B)$, with its usual dg Lie algebra structure, and we let $L(B ; E)$ denote the $E$-invariants of the shifted, normalized, braided Hochschild complex $\Sigma \bar{C}_{c}^{\bullet}(B)^{E}$. One can see easily that the differential on invariant (i.e. left $E$-linear) functions in $\bar{C}_{c}^{\bullet}(B)$ reduces to the usual, nonbraided, Hochschild differential and that the inclusion

$$
\bar{C}_{c}^{\bullet}(B)^{E} \rightarrow \bar{C}^{\bullet}(B)
$$

induces a dg Lie structure on the shifted complex $L(B ; E)$. The inclusion identifies the invariant functions in $\bar{C}_{c}^{\bullet}(B)$ with left $E$-linear functions in $\bar{C} \bullet(B)$.

Theorem 4.8. Suppose $E$ is both semisimple and cosemisimple and take $B=A * E$. The inclusion $L(B ; E) \rightarrow L(B)$ is a quasi-isomorphism of $d g$ Lie algebras.

Without getting completely sidetracked by the issue, the above corollary says that when $E$ is semisimple and cosemisimple the formal deformation theory of $B$ as an associative algebra, and $B$ as an $E$-module algebra, will be equivalent. See $[19,16,24,33,8,18,6]$.

We note that our complex $L(B ; E)$ representes the formal deformation theory of $B$ with fixed $E$-module structure. However, since $E$ is semisimple $B$ will not be deformable as an $E$-module, so the distinction should be immaterial (see also [33]).

Proof of Theorem 4.8. In this proof we take $C_{*}^{\bullet}$ to mean the normalized complex, to ease notation. We have the quasi-isomorphism of complexes $C^{\bullet}(A, B)=C_{c, E}^{\bullet}(B) \rightarrow C_{c}^{\bullet}(B)$ and taking invariants gives another quasiisomorphism $C^{\bullet}(A, B)^{E} \rightarrow C_{c}^{\bullet}(B)^{E}$ (since $E$ is semisimple and the invariants functor is therefore exact). So, to see that the shift of the map $C_{c}^{\bullet}(B)^{E} \rightarrow C^{\bullet}(B)$ is a quasi-isomorphism it suffices to show that the composite map $C^{\bullet}(A, B)^{E} \rightarrow C_{c}^{\bullet}(B)^{E} \rightarrow C^{\bullet}(B)$ is a quasi-isomorphism.

But now $C^{\bullet}(A, B)^{E}$ is canonically identified with the relative Hochschild complex $C_{E}^{\bullet}(B)$. Since $E$ is separable it then follows from [10] that the inclusion

$$
\Sigma C^{\bullet}(A, B)^{E}=\Sigma C_{E}^{\bullet}(B) \rightarrow \Sigma C^{\bullet}(B)
$$

is in fact a quasi-isomorphism. 
4.2. Cohomology for crossed products and $J$-twists. Take $E$ a finite dimensional Hopf algebra and $B=A * E$. Let $J \in E^{*} \otimes E^{*}$ be an invertible dual cocycle (or, twist) [22]. We can consider this element also as an element in the second tensor power of the double $D \otimes D$. For any such $J$, and (dg) algebra $\Omega$ in $Y D_{E}^{E}=D \bmod$ we can form the $(\mathrm{dg})$ algebra $\Omega_{J}$ by altering the multiplication

$$
\omega \cdot{ }_{J} \omega^{\prime}=\left(J_{l} \cdot \omega\right)\left(J^{l} \cdot \omega^{\prime}\right)
$$

where $J=J_{l} \otimes J^{l}$. (There is an implicit sum here $J=\sum_{l} J_{l} \otimes J^{l}$.) This new object will be a (dg) algebra in the category of modules over the twists $D^{J}[22]$, and the Hopf algebra $D^{J}$ will be quasitriangular with new $R$-matrix $R=J_{21}^{-1} R J[7]$. So, the algebra $\Omega_{J}$ is an algebra in the braided category $\operatorname{dg} D^{J} \bmod$.

In the case that $E$ is cocommutative (e.g. a group algebra) we arrive at all crossed products $A *_{\alpha} E$, with $\alpha$ taking values in $k$, as $(A * E)_{J}$ where $J$ is the element identified with $\alpha$ under the isomorphism $E^{*} \otimes E^{*} \cong(E \otimes E)^{*}$. So we see that many crossed products of interest live naturally in a braided monoidal categories as well. For example, we can consider the motivating examples of [4]. I make the following claim.

Claim 4.9. Take $B=(A * E)$ and $J$ an invertible dual cocycle in $E^{*} \otimes E^{*} \subset$ $D \otimes D$. Then there is a natural identification

$$
H H^{\bullet}\left(A, B_{J}\right)=H_{c, E_{J}}^{\bullet}\left(B_{J}\right)
$$

and when $E$ is both semisimple and cosemisimple we get a natural isomorphism with the non-relative braided cohomology

$$
H H^{\bullet}\left(A, B_{J}\right) \cong H_{c}^{\bullet}\left(B_{J}\right) .
$$

Whence the Hochschild cohomology has the structure of an object in $\operatorname{dg} D^{J} \bmod$.

With the claim one can provide an analog of Theorem 4.8 for certain crossed products. At this point the proof of the claim is quite arduous (although it can be presented clearly). So we forgo this path and opt instead for a happy in between with the following proposition.

Proposition 4.10. Take $B=A * E$ and any $J$ as above.

(1) There is an equality of $H H^{\bullet}(A)$-bimodules $H H^{\bullet}\left(A, B_{J}\right)=H H^{\bullet}(A, B)$, which also holds at the cochain level.

(2) There is an equality of $d g$ algebras

$$
C^{\bullet}(A, B)_{J}=C^{\bullet}\left(A, B_{J}\right)
$$

and also an equality of Hochschild cohomology rings

$$
H H^{\bullet}(A, B)_{J}=H H^{\bullet}\left(A, B_{J}\right) \text {. }
$$

(3) The equality of (2) gives the Hochschild cohomology of $B_{J}$ the natural structure of an object in the category $\mathrm{dg} D^{J} \bmod$.

We adopt some of the notation (but none of the results) of Section 5 for the proof. 
Proof. We prove (1) last, and begin with (2). Note that $A^{\otimes n} \subset B^{\otimes_{E} n}$ is exactly the coinvariants. So $J=J_{l} \otimes J^{l}$ will act trivially on the tensor powers of $A$. This fact also implies that for any $a \in A$, and $b \in B$, we will have $a \cdot{ }_{J} b=a b$ and $b \cdot{ }_{J} a=b a$, so that $B_{J}=B$ as an $A$-bimodule. Thus we have an equality of cochain complexes $C^{\bullet}(A, B)=C^{\bullet}\left(A, B_{J}\right)$.

Take now any functions $f, g \in C^{\bullet}(A, B)=C^{\bullet}\left(A, B_{J}\right)$, and any $x \in A^{\otimes n}$. Then, when we take the product in $C^{\bullet}(A, B)_{J}$ we get

$$
\begin{aligned}
\pm f \cup_{J} g(x) & =\left(J_{l} \cdot f\right)\left(x_{1}\right)\left(J^{l} \cdot g\right)\left(x_{2}\right) \\
& =\left(J_{l 1} \cdot f\left(S\left(J_{l 2}\right) \cdot x_{1}\right)\right)\left(J_{1}^{l} \cdot g\left(S\left(J_{2}^{l}\right) \cdot x_{2}\right)\right) \\
& =\left(J_{l} \cdot f\left(x_{1}\right)\right)\left(J^{l} \cdot g\left(x_{2}\right)\right) \text { since } x_{i} \text { is coinvariant } \\
& =f\left(x_{1}\right) \cdot J g\left(x_{2}\right) .
\end{aligned}
$$

This last expression is equal to the cup product in $C^{\bullet}\left(A, B_{J}\right)$, and we are done. Take cohomology to get the identification for $H H^{\bullet}$.

For $(3)$ we note that $(-)_{J}$ restricts to a functor from $D$-module algebras to $D^{J}$-module algebras. For (1) we note that $H H^{\bullet}(A)$ sits inside of the coinvariants in $H H^{\bullet}(A, B)$. The same statement holds at the cochain level. So whenever $f$ or $g$ is in $C^{\bullet}(A) \subset C^{\bullet}(A, B)=C^{\bullet}\left(A, B_{J}\right)$ we have

$$
f\left(x_{1}\right) \cdot J g\left(x_{2}\right)=\left(J_{l} \cdot f\left(x_{1}\right)\right)\left(J^{l} \cdot g\left(x_{2}\right)\right)=f\left(x_{1}\right) g\left(x_{2}\right),
$$

since $(\epsilon \otimes i d)(J)=(i d \otimes \epsilon)(J)=1$.

The $D^{J}$-module structure arrived at via twisting is the same $D^{J}$-module structure we arrive at by the more robust means suggested in the claim. Namely, if we forget the coproduct on $D^{J}$ and the product on the Hochschild cohomology, we have $H H^{\bullet}\left(A, B_{J}\right)=H H^{\bullet}(A, B)$ as a $D=D^{J}$-module. As a consequence we find that the $E$-action on Hochschild cohomology arrived at via the algebra (but not Hopf) embedding $E^{o p} \rightarrow D^{J}$ does not agree with those considered in $[32,13,12]$. So it seems that we need to leave our setting, and consider [32] independently, in order to produce a spectral sequence relating the cohomology $H_{c}^{\bullet}\left(B_{J}\right)$ to the Hochschild cohomology $H H^{\bullet}\left(B_{J}\right)$. At the moment this point of confusion remains unresolved.

\section{The CUP PRODUCT AND BRAIDED COMMUtATIVITY OF BRAIDED HOCHSCHILD COHOMOLOGY}

In this section we show that the complex $C_{c}^{\bullet}(B)$ and cohomology $H_{c}^{\bullet}(B)$ both admit canonical products, and that the cohomology is a braided commutative algebra under this product. The same will hold for the relative cohomology as well. Applications to smash products are given in Section 6, as well as Corollary 5.14.

5.1. The braided category $\operatorname{dg} \mathscr{Z}$. For any braided tensor category $\mathscr{Z}=$ $H \bmod$ the category $\operatorname{dg} \mathscr{Z}$ of complexes over $\mathscr{Z}$ carries its own braiding, tensor product, and inner homs. The tensor product of objects $M$ and $N$ in $\operatorname{dg} \mathscr{Z}$ is the usual tensor product of complexes

$$
(M \otimes N)^{i}=\oplus_{i_{1}+i_{2}=i} M^{i_{1}} \otimes N^{i_{2}}
$$


with differential

$$
d_{M \otimes N}(m \otimes n)=d_{M}(m) \otimes n+(-1)^{|m|} m \otimes d_{N}(n),
$$

where $m$ and $n$ are assumed to be homogeneous in the above expression. The braiding will be given by the braiding from $\mathscr{Z}$ with the addition of the standard Koszul sign:

$$
c_{M N}^{\mathrm{dg} \mathscr{Z}}: M \otimes N \rightarrow N \otimes M, \quad m \otimes n \mapsto(-1)^{|m||n|} r^{j} \cdot n \otimes r_{j} \cdot n .
$$

The inner homs are the expected hom complexes

$$
\underline{\operatorname{Hom}}(M, N))^{i}=\prod_{j_{2}-j_{1}=i} \underline{\operatorname{Hom}}\left(M^{j_{1}}, N^{j_{2}}\right)
$$

with differential $d_{\underline{\text { Hom }}}(f)=d_{N} f-(-1)^{|f|} f d_{M}$. The action is the action implied by the Hom notation, $(h \cdot f)(m)=h_{1} \cdot\left(f\left(S\left(h_{2}\right) \cdot m\right)\right)$.

The fact that both $d_{M}$ and $d_{N}$ commute with the $H$-action on $M$ and $N$ respectively implies that $d_{\text {Hom }}$ commutes with the action of $H$ as well. One can check easily that the pairing

$$
\underline{\operatorname{Hom}}(M, N) \otimes M \rightarrow N
$$

will always be a map of complexes in $\operatorname{dg} \mathscr{Z}$. We adopt a similar notation for $\underline{\text { Hom }}_{E}$.

In general when doing computations with chain complexes we take all elements to be homogeneous.

5.2. The cup product. Recall that the bar construction

$$
\mathscr{B} B=\cdots \rightarrow B^{\otimes 3} \rightarrow B^{\otimes 2} \rightarrow B \rightarrow k \rightarrow 0
$$

is a dg coalgebra with comultiplication given by separation of tensors. For $x=b_{1} \otimes \ldots \otimes b_{n}$ we have

$$
\Delta_{\mathscr{B} B}(x)=(x) \otimes(1)+(1) \otimes(x)+\sum_{i=1}^{n-1}\left(b_{1} \otimes \ldots \otimes b_{i}\right) \otimes\left(b_{i+1} \otimes \ldots \otimes b_{n}\right) .
$$

Indeed, with the addition of the diagonal $H$-action $\mathscr{B} B$ becomes a dg coalgebra in $\mathscr{Z}=H$ mod.

Lemma 5.1. For any coalgebra $\Gamma$ in $\operatorname{dg} \mathscr{Z}$ and $d g$ algebra $\Omega$ in $\operatorname{dg} \mathscr{Z}$ the inner hom complex $\underline{\operatorname{Hom}}(\Gamma, \Omega)$ becomes an algebra in $\operatorname{dg} \mathscr{Z}$ under the (braided) convolution product

$$
f \cup g(\gamma)=(-1)^{\left|\gamma_{1}\right||g|} f\left(r^{j} \cdot \gamma_{1}\right)\left(r_{j} \cdot g\right)\left(\gamma_{2}\right),
$$

for $f, g \in \underline{\operatorname{Hom}}(\Gamma, \Omega), \gamma \in \Gamma$.

Proof. The verification that the product is compatible with the differential is straightforward and will be omitted. To see that the product is a map in 
$\mathscr{Z}=H \bmod$, note that we arrive at the element $\pm f\left(r^{j} \cdot \gamma_{1}\right)\left(r_{j} \cdot g\right)\left(\gamma_{2}\right)$ via the sequence of maps in $\mathscr{Z}$

$$
\begin{aligned}
& \underline{\mathrm{Hom}}^{\otimes 2} \otimes \Gamma \stackrel{i d \otimes \Delta}{\longrightarrow} \underline{\operatorname{Hom}}^{\otimes 2} \otimes \Gamma \otimes \Gamma \stackrel{i d \otimes c \otimes i d}{\longrightarrow} \underline{\operatorname{Hom}} \otimes \Gamma \otimes \underline{\operatorname{Hom}} \otimes \Gamma \\
& \stackrel{\text { pair } \otimes \text { pair }}{\longrightarrow} \Omega \otimes \Omega \stackrel{\text { mult }}{\longrightarrow} \Omega \text {, }
\end{aligned}
$$

where $\underline{\operatorname{Hom}}=\underline{\operatorname{Hom}}(\Gamma, \Omega)$. So, $H$-linearity tells us that for any $h \in H$, $f, g \in \underline{\operatorname{Hom}}(\Gamma, \Omega)$, and $\gamma \in \Gamma$, we have

$$
\pm h \cdot\left(f\left(r^{j} \cdot \gamma_{1}\right)\left(r_{j} \cdot g\right)\left(\gamma_{2}\right)\right)= \pm\left(h_{1} \cdot f\right)\left(r^{j} h_{3} \cdot \gamma_{1}\right)\left(r_{j} h_{2} \cdot g\right)\left(h_{4} \cdot \gamma_{2}\right)
$$

Replace $h \otimes \gamma_{1} \otimes \gamma_{2}$ with $h_{1} \otimes S\left(h_{3}\right) \gamma_{1} \otimes S\left(h_{2}\right) \gamma_{2}=h_{1} \otimes \Delta\left(S\left(h_{2}\right) \gamma\right)$ to get then

$$
\begin{aligned}
(h \cdot(f \cup g))(\gamma) & = \pm h_{1} \cdot\left(f\left(r^{j} S\left(h_{3}\right) \cdot \gamma_{1}\right)\left(r_{j} \cdot g\right)\left(S\left(h_{2}\right) \cdot \gamma_{2}\right)\right) \\
& = \pm\left(h_{1} \cdot f\right)\left(r^{j} \cdot \gamma_{1}\right)\left(r_{j} h_{2} \cdot g\right)\left(\gamma_{2}\right) \\
& \left.=\left(\left(h_{1} \cdot f\right) \cup\left(h_{2} \cdot g\right)\right)\right)(\gamma) .
\end{aligned}
$$

This verifies that $\underline{\operatorname{Hom}}(\Gamma, \Omega)$ with the proposed product is a, possibly nonassociative, algebra in $\operatorname{dg} \mathscr{Z}$.

For associativity, take $a, b, c \in \underline{\operatorname{Hom}}(\Gamma, \Omega)$. We have

$$
\begin{aligned}
((a \cup b) \cup c)(\gamma) & =a\left(r^{\nu} r_{1}^{j} \cdot \gamma_{1}\right)\left(r_{\nu} \cdot b\right)\left(r_{2}^{j} \cdot \gamma_{2}\right)\left(r_{j} \cdot c\right)\left(\gamma_{3}\right) \\
& =a\left(r^{\nu} r^{j} \cdot \gamma_{1}\right)\left(r_{\nu} \cdot b\right)\left(r^{l} \cdot \gamma_{2}\right)\left(r_{l} r_{j} \cdot c\right)\left(\gamma_{3}\right) \quad \text { (braid relation). }
\end{aligned}
$$

On the other hand we have

$$
\begin{aligned}
(a \cup(b \cup c))(\gamma) & =a\left(r^{\nu} \cdot \gamma_{1}\right)\left(\left(r_{\nu}\right)_{1} \cdot b\right)\left(r^{l} \cdot \gamma_{2}\right)\left(r_{l}\left(r_{\nu}\right)_{2} \cdot c\right)\left(\gamma_{3}\right) \\
& =a\left(r^{\nu} r^{j} \cdot \gamma_{1}\right)\left(r_{\nu} \cdot b\right)\left(r^{l} \cdot \gamma_{2}\right)\left(r_{l} r_{j} \cdot c\right)\left(\gamma_{3}\right) \quad \text { (braid relation). }
\end{aligned}
$$

These expressions are exactly the same, and associativity is verified.

We have an identification of graded objects in $\mathscr{Z}$

$$
C_{c}^{\bullet}(B)=\underline{\operatorname{Hom}}(\mathscr{B} B, B)
$$

and so we get a natural product on $C_{c}^{\bullet}(B)$ induced by the convolution product on $\underline{\operatorname{Hom}}(\mathscr{B} B, B)$. This gives the braided Hochschild complex the structure of an associative algebra in $\mathscr{Z}$, although we've yet to establish compatibility with the differential. In keeping with tradition, we call this product on $C_{c}^{\bullet}(B)$ the (braided) cup product.

Proposition 5.2. The braided Hochschild cochain complex $C_{c}^{\bullet}(B)$, along with the cup product, is an algebra in $\mathrm{dg} \mathscr{Z}$. Furthermore, for any subalgebra $E \subset B$ in $\mathscr{Z}$ the subcomplex $C_{c, E}^{\bullet}(B) \subset C_{c}^{\bullet}(B)$ is a subalgebra in $\operatorname{dg} \mathscr{Z}$.

By an algebra in $\operatorname{dg} \mathscr{Z}$ we mean in particular that it is a dg algebra. In order to prove the proposition it will be helpful to have the following lemma.

Lemma 5.3. The differential on the complex $C_{c}^{\bullet}(B)=\left(\underline{\operatorname{Hom}}(\mathscr{B} B, B), d_{c}\right)$ is the sum

$$
d_{c}=d_{\underline{\operatorname{Hom}}(\mathscr{B} B, B)}-[\pi,-],
$$


where $\pi$ is the degree 1 map $\mathscr{B} B \rightarrow B$ which restricts to the identity on $\mathscr{B} B^{-1}=B$, and $[\pi,-]$ is the usual graded commutator $[\pi, f]=\pi \cup f-$ $(-1)^{|f|} f \cup \pi$.

Proof. Since $\pi$ is a map in $\mathscr{Z}=H \bmod$ we will have $h \cdot \pi=\epsilon(h) \pi$ for each $h \in H$. Consider now a degree $n$ function $f$ and $x=b_{1} \otimes \ldots \otimes b_{n+1}$. Then

$$
\begin{aligned}
& (-1)^{|f|}[\pi, f](x) \\
& =\pi\left(r^{j} \cdot b_{1}\right)\left(r_{j} \cdot f\right)\left(b_{2} \otimes \ldots b_{n+1}\right)-(-1)^{n} f\left(b_{1} \otimes \ldots \otimes b_{n}\right) \pi\left(b_{n+1}\right) \\
& =\left(r^{j} \cdot b_{1}\right)\left(r_{j} \cdot f\right)\left(b_{2} \otimes \ldots b_{n+1}\right)-(-1)^{n} f\left(b_{1} \otimes \ldots \otimes b_{n}\right) b_{n+1} \\
& =(-1)^{|f|+1} d_{c}(f)(x)-f\left(d_{\mathscr{B} B}(x)\right) \\
& =(-1)^{|f|+1}\left(d_{c}(f)-d_{\text {Hom }}(f)\right)(x) .
\end{aligned}
$$

This implies

$$
-[\pi, f]=d_{c}(f)-d_{\underline{\mathrm{Hom}}}(f) \Rightarrow d_{c}(f)=d_{\underline{\mathrm{Hom}}}(f)-[\pi, f] .
$$

Proof of Proposition 5.2. As noted above, $\pi$ is $H$-invariant so that, by the relation $(1 \otimes \epsilon)\left(R_{21}\right)=1 \otimes 1$ the cup product of $\pi$ with itself will be given by the non-braided formula

$$
(\pi \cup \pi)(x)=\pi\left(r^{j} \cdot x_{1}\right)\left(r_{j} \cdot \pi\right)\left(x_{2}\right)=\pi\left(x_{1}\right) \pi\left(x_{2}\right) .
$$

Whence we see that on degree -2 elements we have $(\pi \cup \pi)\left(b \otimes b^{\prime}\right)=b b^{\prime}$ and find that $\pi$ solves the Maurer-Cartan equation

$$
d_{\underline{\text { Hom }}}(\pi)-\pi \cup \pi=0
$$

in the dg algebra $\underline{\operatorname{Hom}}(\mathscr{B} B, B)$. It is then standard that the new object

$$
\left(\underline{\operatorname{Hom}}(\mathscr{B} B, B), d_{\text {Hom }}-[\pi,-]\right)=C_{c}^{\bullet}(B)
$$

is a dg algebra under the same product as $\underline{\operatorname{Hom}}(\mathscr{B} B, B)[17]$.

As for the fact that $C_{c, E}^{\bullet}(B)$ is a subalgebra in $C_{c}^{\bullet}(B)$, we already know that it is a subcomplex in $\operatorname{dg} \mathscr{Z}$. So we need only see that it is a subalgebra under the cup product. We have for $w \in E, x \in \mathscr{B} B$, and $f, g \in C_{c, E}^{\bullet}(B)$,

$$
\begin{aligned}
(f \cup g)(w x) & =f\left(r^{j} \cdot\left(w x_{1}\right)\right)\left(r_{j} \cdot g\right)\left(x_{2}\right) \\
& =\left(r^{k} r^{j} \cdot w\right)\left(r_{k} \cdot f\right)\left(r^{l} \cdot x_{1}\right)\left(r_{l} r_{j} \cdot g\right)\left(x_{2}\right) \\
& =\left(r^{j} \cdot w\right)\left(\left(r_{j}\right)_{1} \cdot f\right)\left(r^{l} \cdot x_{1}\right)\left(r_{l}\left(r_{j}\right)_{2} \cdot g\right)\left(x_{2}\right) \\
& =\left(r^{j} \cdot w\right)\left(\left(\left(r_{j}\right)_{1} \cdot f\right) \cup\left(\left(r_{j}\right)_{2} \cdot g\right)\right)(x) \\
& =\left(r_{j} \cdot w\right)\left(r_{j} \cdot(f \cup g)\right)(x) .
\end{aligned}
$$

One checks by a similar application of (2.2) that

$$
f\left(r^{j} \cdot x_{1}\right)\left(r_{j} \cdot g\right)\left(w x_{2}\right)=f\left(r^{j} \cdot\left(x_{1} w\right)\right)\left(r_{j} \cdot g\right)\left(x_{2}\right),
$$

and right $E$-linearity $f \cup g(x w)=f \cup g(x) w$ follows by right $E$-linearity of $g$. One can check easily from these three relations that $f \cup g$ satisfies all the relations necessary so that it lay in the subcomplex $\underline{\operatorname{Hom}}_{E}\left(\mathscr{B}_{E} B, B\right)=$ $C_{c, E}^{\bullet}(B) \subset C_{c}^{\bullet}(B)$.

Taking cohomology then implies, from Proposition 5.2, 
Corollary 5.4. (1) The braided Hochschild cohomology $H_{c}^{\bullet}(B)$, as well as its relative versions $H_{c, E}^{\bullet}(B)$, are all algebras is $\operatorname{dg} \mathscr{Z}$ (with vanishing differential).

(2) There are canonical algebra maps $H_{c, E}^{\bullet}(B) \rightarrow H_{c}^{\bullet}(B)$ in $\operatorname{dg} \mathscr{Z}$.

(3) When $E$ is semisimple and cosemisimple, $\mathscr{Z}=Y D_{E}^{E}$, and $B=A * E$, the algebra map $H_{c, E}^{\bullet}(B) \rightarrow H_{c}^{\bullet}(B)$ is an algebra isomorphism in $\operatorname{dg} \mathscr{Z}$.

Proof. (1) and (2) are consequences of Proposition 5.2. For (3) we consider also Theorem 4.5 .

Since $\mathscr{B} A=(\mathscr{B} B)^{c o E}$ when $B=A * E$, we get that the braided cup product of functions $f \cup g$ restricted to $\mathscr{B} A$ recovers the standard cup product on the Hochschild cochain complex, under the identification $C_{c, E}^{\bullet}(B)=$ $C^{\bullet}(A, B)$ of Proposition 4.2. Whence we also get

Corollary 5.5. When $B=A * E$

(1) there identification $H H^{\bullet}(A, B)=H_{c, E}^{\bullet}(B)$ of Proposition 4.2 is one of graded algebras.

(2) The standard Hochschild cohomology $H^{\bullet}(A, B)$ is an algebra in $\operatorname{dg} Y D_{E}^{E}$.

(3) There is a multiplicative spectral sequence

$$
\operatorname{Ext}_{\bmod E}\left(k, H_{c, E}^{\bullet}(B)\right) \Rightarrow H H^{\bullet}(B) .
$$

When, additionally, $E$ is semisimple and cosemisimple

(4) there is an algebra identification $H H^{\bullet}(A, B)=H_{c}^{\bullet}(B)$ in $\operatorname{dg} Y D_{E}^{E}$.

(5) There is an algebra identification $H H^{\bullet}(B)=H_{c}^{\bullet}(B)^{E}$.

Proof. Statement (1) is clear from the discussion preceding this corollary. (2) follows from (1). (3) follows from the preexisting spectral sequence

$$
\operatorname{Ext}_{\bmod E}\left(k, H H^{\bullet}(A, B)\right) \Rightarrow H H^{\bullet}(B)
$$

from [25]. (4) follows from (1) and Corollary 5.4. (5) follows from (3), as the spectral sequence will collapse. (5) can also be deduced from [10].

We can also twist by a dual cocycle to get

Corollary 5.6. Take $B=A * E$. In the presence of an invertible dual cocycle $J \in E^{*} \otimes E^{*}$ the algebra $H H^{\bullet}\left(A, B_{J}\right)$ is an algebra in the braided category $\operatorname{dg} D^{J} \bmod$.

Proof. The result follows from Proposition 4.10 and the fact that the $J$-twist sends dg algebras in Dmod to dg algebras in $D^{J} \bmod [22]$.

We presumably will not get an identification of algebras $H H^{\bullet}(B)=$ $H_{c, E}^{\bullet}(B)^{E}$ if we include a dual cocycle $J$, since $E$ is not a Hopf subalgebra of the twisted double in general, and so $C_{c, E_{J}}^{\bullet}\left(B_{J}\right)$ will not be a dg algebra in Emod. So it makes no sense to think about the " $E$-invariant subalgebra" 
here. This brings us back to the concerns expressed at the conclusion of Section 4.2.

Remark 5.7. Although we have ignored the issue, our production of a product on the cohomology $H_{c}^{\bullet}(B)$ is somewhat curious, since the identification

$$
H_{c}^{\bullet}(B)=\operatorname{Ext}_{\bmod B} \underline{e}(B, B)
$$

tells us that this object already has a natural product (the Yoneda product). This curiosity also appears in the non-braided setting with the production of the usual cup product. We expect that, as in the non-braided setting, the two products will agree. Namely, we should be able to produce a dg algebra quasi-isomorphism

$$
C_{c}^{\bullet}(B) \rightarrow \operatorname{End}_{\bmod B \underline{e}}\left(\mathscr{B} a r^{c} B, \mathscr{B} a r^{c} B\right)
$$

just as in [23]. One should simply apply the braiding where appropriate.

\subsection{Braided commutativity.}

Definition 5.8. We define the circle operation

$$
\circ: C_{c}^{\bullet}(B) \otimes C_{c}^{\bullet}(B) \rightarrow C_{c}^{\bullet}(B)
$$

by

$$
f \circ g(x)=(-1)^{\left|x_{1}\right|(|g|-1)} f\left(\left(r^{j} \cdot x_{1}\right) \otimes\left(r_{j} \cdot g\right)\left(x_{2}\right) \otimes x_{3}\right)
$$

and the naïve bracket by

$$
[f, g]_{\circ}=f \circ g-(-1)^{(|f|-1)(|g|-1)}\left(r^{j} \cdot g\right) \circ\left(r_{j} \cdot f\right) .
$$

Here we've used the identifications $B^{\otimes n_{1}} \otimes B^{\otimes n_{2}} \otimes B^{\otimes n_{3}}=B^{\otimes n_{1}+n_{2}+n_{3}}$, and subsequent concatenation map $\mathscr{B} B \otimes \mathscr{B} B \otimes \mathscr{B} B \rightarrow \mathscr{B} B$ to view the elements $r^{j} \cdot x_{1} \otimes\left(r_{j} \cdot g\right)\left(x_{2}\right) \otimes x_{3}$ as laying in the bar construction of $B$. One subtlety of this point is that, when $x_{1}$ is in $B^{\otimes 0}=k$, for example, the element $r^{j} \cdot x_{1} \otimes\left(r_{j} \cdot g\right)\left(x_{2}\right) \otimes x_{3}$ is identified with $g\left(x_{1}\right) \otimes x_{2}$. Save for the presence of $R_{21}$, this formula looks exactly like the usual circle operation [9], and can be identified with composition of braided coderivations as in [31].

The bracket [,]。 will not preserve cocycles or coboundaries in general. One needs to do a bit more work in order to produce a well behaved bracket operation which extends the Gerstenhaber bracket to the braided setting (see [24, Ch. 3]). This poorly behaved bracket will, however, still prove quite useful.

By the usual computations one checks

Lemma 5.9. For any subalgebra $E \subset B$ the dg subalgebra $C_{c, E}^{\bullet}(B) \subset C_{c}^{\bullet}(B)$ is preserved by the circle operation and naïve bracket.

We can now prove an essential technical result.

Proposition 5.10. For $f, g$ in $C_{c}^{\bullet}(B)$ or $C_{c, E}^{\bullet}(B)$ we have

$$
\begin{aligned}
& (-1)^{|f|+1} d_{c}(f \circ g)+(-1)^{|f|} d_{c}(f) \circ g-f \circ d_{c}(g) \\
& =f \cup g-(-1)^{|f||g|}\left(r^{j} \cdot g\right) \cup\left(r_{j} \cdot f\right) .
\end{aligned}
$$


In particular, if $f$ and $g$ are cocycles then

$$
(-1)^{|f|+1} d(f \circ g)=f \cup g-(-1)^{|f||g|}\left(r^{j} \cdot g\right) \cup\left(r_{j} \cdot f\right) .
$$

Proof. This is just as in [9]. Let $\mu: \mathscr{B}_{E} B \rightarrow B$ be the degree 2 function given on $B^{\otimes_{E} 2}$ by the multiplication $b \otimes b^{\prime} \mapsto b b^{\prime}$, and 0 on all other tensor powers. Then the differential $d_{c}$ is exactly the naïve bracket $d_{c}(f)=[\mu, f]_{\circ}$. With this interpretation one checks the identity

$$
\begin{aligned}
& {[\mu, f \circ g]_{\circ}} \\
& =[\mu, f]_{\circ} \circ g-(-1)^{|f||g|+|f|-1}\left(r_{j} \cdot g\right) \cup\left(r_{j} \cdot f\right)-(-1)^{|f|} f \cup g-(-1)^{|f|} f \circ[\mu, g]_{\circ} .
\end{aligned}
$$

Rearranging gives

$$
\begin{aligned}
& {[\mu, f \circ g]_{\circ}-[\mu, f]_{\circ} \circ g+(-1)^{|f|} f \circ[\mu, g]_{\circ}} \\
& =(-1)^{|f|+1} f \circ[\mu, g]_{\circ}-(-1)^{|f||g|+|f|-1} r_{j} g \cup r_{j} f .
\end{aligned}
$$

Multiplying by $(-1)^{|f|+1}$ and replacing $[\mu,-]$ with $d_{c}$ gives the formula (5.2).

We call an algebra $\Omega$ in $\operatorname{dg} \mathscr{Z}$ commutative in $\operatorname{dg} \mathscr{Z}$, or braided commutative, if for each homogeneous $\omega, \omega^{\prime} \in \Omega$ we have

$$
\omega \omega^{\prime}-(-1)^{|\omega|\left|\omega^{\prime}\right|}\left(r^{j} \cdot \omega^{\prime}\right)\left(r_{j} \cdot \omega\right)=0 .
$$

This notion is not new [1].

Theorem 5.11. The braided cohomologies $H_{c}^{\bullet}(B)$, as well as the relative versions $H_{c, E}^{\bullet}(B)$, are commutative algebras in $\operatorname{dg} \mathscr{Z}$.

Proof. Equation (5.3) of Proposition 5.10 implies that the braided commutator of any two cocycles is a coboundary.

Remark 5.12. One can compare Theorem 5.11, and its proof, to [20, Corollary 3.13]. In our language, the "Hochschild cohomology" considered in [20] should be the braided Hochschild cohomology with coefficients in the unit $\mathbf{1}_{\mathscr{Z}}$.

The first portion of the next result was deduced independently in [28] in the case of a finite group acting in characteristic 0 .

Corollary 5.13. For $B=A * E$ the cohomology $H H^{\bullet}(A, B)$ is braided commutative in $\operatorname{dg} Y D_{E}^{E}$. For any invertible dual cocycle $J$, the cohomology $H H^{\bullet}\left(A, B_{J}\right)$ is braided commutative in $\operatorname{dg} D^{J}$.

Proof. The result for $H H^{\bullet}(A, B)$ follows from the algebra identification $H H^{\bullet}(A, B)=H_{c, E}^{\bullet}(B)$ of Corollary 5.5. In the presence of the twist $J$, the result follows from Proposition 4.10 and the fact that the twist functor $(-)_{J}$ sends commutative algebras in $\mathrm{dg} D \bmod$ to commutative algebras in $\operatorname{dg} D^{J} \bmod$.

The following corollary seems to be new. We let $\operatorname{Center}(\Omega)$ denote the usual graded center of a dg algebra $\Omega$. 
Corollary 5.14. When $E$ is semisimple and $B=A * E$, there is an algebra inclusion

$$
H H^{\bullet}(B) \subset \operatorname{Center}\left(H H^{\bullet}(A, B)\right) .
$$

Proof. Recall $H H^{\bullet}(B)=H H^{\bullet}(A, B)^{E}$. Since $H H^{\bullet}(A, B)$ is braided commutative, we have $\bar{f} \cup \bar{g}= \pm\left(r^{j} \cdot \bar{g}\right) \cup\left(r_{j} \cdot \bar{f}\right)$ for any classes $\bar{f}, \bar{g} \in H H^{\bullet}(A, B)$. Thus, when $\bar{f}$ is invariant we have

$$
\bar{f} \cup \bar{g}= \pm\left(r^{j} \cdot \bar{g}\right) \cup\left(r_{j} \cdot \bar{f}\right)= \pm\left(r^{j} \cdot \bar{g}\right) \cup\left(\epsilon\left(r_{j}\right) \bar{f}\right)= \pm \bar{g} \cup \bar{f} .
$$

So invariant functions are central and we get the proposed embedding, since $H H^{\bullet}(B)$ is identified with the invariants in $H H^{\bullet}(A, B)=H H_{c}^{\bullet}(B)$.

In general, we don't expect $H H^{\bullet}(B)$ to be the entire center. For example, in the case of a finite group $G$ acting faithfully by linear automorphisms on the polynomials ring $S(V)$ generated by a vector space $V$, the cohomology $H H^{\bullet}(S(V), S(V) * G)$ in degree 0 is just $S(V)$. One can then check that all the elements in $H H^{0}(S(V), S(V) * G)=S(V)$ are already central in $H H^{\bullet}(S(V), S(V) * G)$, despite the fact that $S(V)^{G}$ is not equal to $S(V)$. One can also see this from Proposition 6.1 below.

\section{Consequences for smash products with group algebras}

Fix a finite group $G$ acting on an algebra $A$. We consider only the smash product $A * G$, although one could just as easily consider a crossed product $A *_{\alpha} G$, or $J$-twist, according to Proposition 4.10. As has been our general convention, we let $G^{o p} \subset D$ act on the left of Yetter-Drinfeld modules (opposed to $G$ acting on the right) as described in 2.3.

Recall that we have the $A$-bimodule decomposition $A * G=\oplus_{g \in G} A g$. This gives a canonical decomposition

$$
H_{c, k G}^{\bullet}(A * G)=H H^{\bullet}(A, A * G)=\bigoplus_{g \in G} H H^{\bullet}(A, A g),
$$

which, as we've mentioned before, expresses the canonical $G$-grading ( $G$ coaction) on the dg Yetter-Drinfeld module $H_{c, k G}^{\bullet}(A * G)$. Note that the usual Hochschild cohomology $H H^{\bullet}(A, A)=H H^{\bullet}(A, A e)$ sits inside the cohomology $H H^{\bullet}(A, A * G)$, and hence $H_{c, k G}^{\bullet}(A * G)$, as the graded subalgebra of coinvariants. The cohomology $H H^{\bullet}(A)$ then acts naturally on each component $H H^{\bullet}(A, A g)$ by the cup product.

Proposition 6.1. Suppose $G$ is a finite group and, for each $g \in G$, let $I_{g}$ be the ideal in $H H^{\bullet}(A)$ generated by all the classes $(1-g) \cdot Y$, with $Y \in H H^{\bullet}(A)$. Each $H H^{\bullet}(A)$-module $H H^{\bullet}(A, A g)$ is annihilated by $I_{g}$, both on the left and the right. Furthermore, each $H H^{\bullet}(A, A g)$ is a central bimodule over $H H^{\bullet}(A) / I_{g}$ and $H H^{\bullet}(A)$.

Proof. For any $Y_{e} \in H H^{\bullet}(A)$ and $Y_{g} \in H H^{\bullet}(A, A g)$ braided commutativity gives

$$
Y_{e} Y_{g}=Y_{g}\left(Y_{e} \cdot g\right)=\left(g \cdot Y_{e}\right) Y_{g} \Rightarrow\left((1-g) \cdot Y_{e}\right) Y_{g}=0 .
$$


Hence the cohomology is annihilated by each $(1-g) \cdot Y_{e}$ and therefore also the ideal $I_{g}$. A similar computation holds on the right as well. The fact that $H H^{\bullet}(A) / I_{g}$ acts centrally on $H H^{\bullet}(A, A g)$ follows from the fact that the induced $g$-action on the quotient $H H^{\bullet}(A) / I_{g}$ is trivial. Now we see that $H H^{\bullet}(A)$ also acts centrally, as the right and left actions factor through the quotient $H H^{\bullet}(A) / I_{g}$.

The ideals $I_{g}$ will be quite large in general. In Section 6.1 we explain what work these ideals do in a geometric setting.

Although we leave our investigation here, we remark that braided commutativity will also produce certain generic relations for the Hochschild cohomology of other smash products $A * E$, e.g. when $E$ is a restricted enveloping algebra. These relations are not quite as straightforward to analyze as in the case of a group ring.

6.1. Understanding the ideals $I_{g}$ from a geometric perspective. Suppose $\operatorname{char}(k)=0$ and let $X$ be a smooth affine scheme over $k$. Fix also a finite group $G$ acting on $X$. We let $k[X]$ denote the algebra of global functions on $X$. In this case the HKR theorem tells us that

$$
H H^{\bullet}(k[X])=\bigwedge_{k[X]}^{\bullet} T_{X},
$$

where $T_{X}$ is the module of global vector fields on $X$ (or, derivations on $k[X])$. Take $X^{g}$ to be the fixed subscheme for a given $g \in G$.

In this case each ideal $I_{g}$ will be generated in degrees 0 and 1 . We will have

$$
H H^{0}(k[X]) / I_{g}^{0}=k[X] / I_{g}^{0}=k[X] /\left(f-{ }^{g} f\right)_{f \in k[X]}=k\left[X^{g}\right],
$$

and for the whole cohomology there is a canonical algebra identification

$$
H H^{\bullet}(k[X]) / I_{g}=\wedge_{k\left[X^{g}\right]}^{\bullet}\left(T_{X}\right) \mid X^{g} /(Y-g \cdot Y)_{|Y|=1}=\bigwedge_{k\left[X^{g}\right]}^{\bullet} T_{X^{g}}
$$

Proposition 6.1 then tells us that the algebra of polyvector fields for each fixed space $X^{g}$ will act naturally on the cohomology $H H^{\bullet}(k[X], k[X] g)$. Each of these actions is defined canonically and globally.

In the case in which $X=\mathbb{A}_{k}^{n}$ and $G$ acts by linear automorphisms we understand that

(a) the ideal $I_{g}$ is the entire annihilator of each $H H^{\bullet}(k[X], k[X] g)$ and

(b) each cohomology $H H^{\operatorname{codim} X^{g}}(k[X])$ is a free rank $1 k\left[X^{g}\right]$-module, let's call it $L_{g}$, and the restriction of the action map

$$
\bigoplus_{g \in G} \bigwedge_{k\left[X^{g}\right]}^{\bullet} T_{X^{g}} \otimes_{k\left[X^{g}\right]} L_{g} \rightarrow H H^{\bullet}(k[X], k[X] * G)=H_{c}^{\bullet}(k[X] * G)
$$

is a $H H^{\bullet}(k[X])$-bimodule isomorphism.

This follows by the careful analysis of [29], for example. Both (a) and (b) are also known to hold when we replace $k[X]$ with smooth functions on a manifold $[26,14]$. In the case $X=\mathbb{A}_{k}^{n}$ the $L_{g}$ form their own subalgebra in the cohomology $k[X]\left[L_{g}: g \in G\right]$ (basically the volume subalgebra in [29]). 
This subalgebra, along with the $H H^{\bullet}(A)$-action, which we've described in general, give the entire algebra structure on $H^{\bullet}(k[X], k[X] * G)=$ $H_{c}^{\bullet}(k[X] * G)$. One then takes invariance to get the cup product on the usual Hochschild cohomology $H H^{\bullet}(k[X] * G)$.

It seems that the above statements will hold for an arbitrary smooth affine $G$-scheme $X$, where we propose now that the $L_{g}$ are locally free. However, the details have yet to emerge in the literature.

\section{ACKNowledgements}

Thanks to Travis Schedler and Sarah Witherspoon for agreeing to share their unpublished notes [28], and for helpful conversations in general. Thanks also to Richard $\mathrm{Ng}$, who has had an influence on my thinking about braided categories.

\section{REFERENCES}

[1] J. C. Baez. Hochschild homology in a braided tensor category. Transactions of the American Mathematical Society, 344(2):885-906, 1994.

[2] V. Baranovsky. Orbifold cohomology as periodic cyclic homology. International Journal of Mathematics, 14(08):791-812, 2003.

[3] J. Brodzki, S. Dave, and V. Nistor. The periodic cyclic homology of crossed products of finite type algebras. preprint arXiv:1509.03662, 2015.

[4] A. Căldăraru, A. Giaquinto, and S. Witherspoon. Algebraic deformations arising from orbifolds with discrete torsion. Journal of Pure and Applied Algebra, 187(1):51-70, 2004.

[5] V. Dolgushev and P. Etingof. Hochschild cohomology of quantized symplectic orbifolds and the Chen-Ruan cohomology. International Mathematics Research Notices, 2005(27):1657-1688, 2005.

[6] V. A. Dolgushev and C. L. Rogers. A version of the Goldman-Millson theorem for filtered $l_{\infty}$-algebras. Journal of Algebra, 430:260-302, 2015.

[7] P. Etingof, S. Gelaki, D. Nikshych, and V. Ostrik. Tensor Categories, volume 205. American Mathematical Society, 2015.

[8] Y. Frégier, M. Markl, and D. Yau. The l-deformation complex of diagrams of algebras. New York Journal of Mathematics, 15:353-392, 2009.

[9] M. Gerstenhaber. The cohomology structure of an associative ring. Annals of Mathematics, pages 267-288, 1963.

[10] M. Gerstenhaber and S. D. Schack. Relative hochschild cohomology, rigid algebras, and the bockstein. Journal of Pure and Applied Algebra, 43(1):53-74, 1986.

[11] V. Ginzburg. Lectures on noncommutative geometry. online arXiv:math/0506603, 2005.

[12] J. Guccione and J. Guccione. Hochschild (co) homology of Hopf crossed products. K-theory, 25(2):139-169, 2002.

[13] A. Guichardet. Suites spectrales à la Hochschild-Serre pour les produits croisés d'algèbres et de groupes. Journal of Algebra, 235(2):744-765, 2001.

[14] G. Halbout and X. Tang. Noncommutative poisson structures on orbifolds. Transactions of the American Mathematical Society, 362(5):2249-2277, 2010.

[15] A. Kaygun. Hopf-Hochschild (co) homology of module algebras. Homology, Homotopy and Applications, 9(2):451-472, 2007.

[16] M. Kontsevich and Y. Soibelman. Deformation theory. Livre en préparation. 
[17] J.-L. Loday and B. Vallette. Algebraic operads, volume 346 of Grundlehren der Mathematischen Wissenschaften [Fundamental Principles of Mathematical Sciences]. Springer, Heidelberg, 2012.

[18] J. Lurie. Derived algebraic geometry x: Formal moduli problems. preprint math.harvard.edu/lurie/papers/DAG-X.pdf.

[19] M. Manetti. Deformation theory via differential graded lie algebras. online arXiv:math/0507284, 2005.

[20] M. Mastnak, J. Pevtsova, P. Schauenburg, and S. Witherspoon. Cohomology of finitedimensional pointed Hopf algebras. Proceedings of the London Mathematical Society, 100(3):377-404, 2010.

[21] S. Montgomery. Hopf algebras and their actions on rings, volume 82 of CBMS Regional Conference Series in Mathematics. Published for the Conference Board of the Mathematical Sciences, Washington, DC, 1993.

[22] S. Montgomery. Algebra properties invariant under twisting. Hopf Algebras in Noncommutative Geometry and Physics. Lecture Notes in Pure and Appl. Math, 239:229243, 2004.

[23] C. Negron. The cup product on Hochschild cohomology via twisting cochains and applications to Koszul rings. preprint arXiv:1304.052\%.

[24] C. Negron. Alternate Approaches to the Cup Product and Gerstenhaber Bracket on Hochschild Cohomology. PhD thesis, University of Washington, 2015.

[25] C. Negron. Spectral sequences for the cohomology rings of a smash product. Journal of Algebra, 433:73-106, 2015.

[26] N. Neumaier, M. J. Pflaum, H. Posthuma, and X. Tang. Homology of formal deformations of proper étale lie groupoids. Journal fur die reine und angewandte Mathematik (Crelles Journal), 2006(593):117-168, 2006.

[27] M. Pflaum, H. Posthuma, X. Tang, and H.-H. Tseng. Orbifold cup products and ring structures on hochschild cohomologies. Communications in Contemporary Mathematics, 13(01):123-182, 2011.

[28] T. Schedler and S. Witherspoon. unpublished notes.

[29] A. V. Shepler and S. Witherspoon. Finite groups acting linearly: Hochschild cohomology and the cup product. Advances in Mathematics, 226(4):2884-2910, 2011.

[30] A. V. Shepler and S. Witherspoon. Group actions on algebras and the graded Lie structure of Hochschild cohomology. Journal of Algebra, 351:350-381, 2012.

[31] J. Stasheff. The intrinsic bracket on the deformation complex of an associative algebra. Journal of pure and applied Algebra, 89(1):231-235, 1993.

[32] D. Stefan. Hochschild cohomology on Hopf Galois extensions. Journal of Pure and Applied Algebra, 103(2):221-233, 1995.

[33] D. Yau. Deformation bicomplex of module algebras. Homology, Homotopy and Applications, 10(1):97-128, 2008.

Department of Mathematics, Louisiana State University, Baton Rouge, LA 70803, USA

E-mail address: cnegron@lsu.edu 